\title{
Validación del Modelo Unidimensional de la Escala de Valoración de la Relación en Personas Casadas y en Unión Libre de Monterrey, México
}

\section{Validation of the Single-Factor Model of the Relationship Assessment Scale among Married and Cohabiting Persons from Monterrey, Mexico}

\author{
Dr. José Moral de la Rubia
}

Universidad Autónoma de Nuevo León

\section{Nota sobre el autor}

Dr. José Moral de la Rubia. Facultad de Psicología. Universidad Autónoma de

Nuevo León

Esta investigación fue financiada con la ayuda del Programa de Apoyo a la Investigación Científica y Tecnológica (PAICYT) de la Universidad Autónoma de Nuevo León (UANL), México. Clave de proyecto: CS454-14.

Remita cualquier duda sobre este artículo al siguiente domicilio: c/Dr. Carlos Canseco 110. Col. Mitras Centro. C.P. 64460. Monterrey, NL, México. Correo electrónico: jose_moral@hotmail.com, jose.morald@uanl.edu.mx 


\section{Resumen}

El estudio de las relaciones de pareja cobra especial importancia, ya que esta unión constituye la base de la familia. La satisfacción con la relación se puede definir como la actitud global hacia la relación y la pareja. La escala de Valoración de la Relación (RAS) de Hendrick es un instrumento comúnmente usado para evaluar el constructo. Esta escala ha demostrado tener consistencia interna alta y una estructura unidimensional. Aunque existen estudios de validación de la RAS, estos han empleado técnicas estadísticas inadecuadas para analizar sus ítems tipo Likert y para determinar el número de factores; además, previamente no se ha contrastado su invarianza factorial por sexo. Por tanto, en este estudio se plantean las siguientes preguntas de investigación: ¿la RAS tiene ítems consistentes y discriminativos? Basados los análisis en la matriz de correlaciones policóricas, ¿cuál es su nivel de consistencia interna?, ¿cuál es su número de factores usando métodos empíricos rigurosos?, y ¿el modelo de un factor es invariante por sexo? A fin de responder a estas preguntas de investigación, se usó un muestreo probabilístico de rutas aleatorias en este estudio instrumental de validación de la RAS. La muestra se extrajo de la población de personas casadas o en unión libre de la ciudad de Monterrey en México. Hubo 431 participantes femeninas y 376 participantes masculinos en este estudio. Los siete ítems de la RAS fueron consistentes y discriminativos. La consistencia interna de la escala fue excelente en la muestra conjunta $(\alpha$ ordinal $=.93)$, en la de mujeres $(\alpha$ ordinal $=.94)$ y en la de hombres $(\alpha$ ordinal $=.92)$. El análisis paralelo de Horn y la prueba de la correlación parcial media mínima de Velicer sugirieron una solución de un factor. Además, el modelo de un factor (con una correlación entre los residuos de los dos ítems inversos) tuvo buen ajuste a los datos y sus propiedades de invarianza por sexo fueron aceptables por el método 
de Mínimos Cuadrados No Ponderados. Se concluye que la escala presenta consistencia interna y validez cruzada por sexo. Se recomienda el uso de la escala en la población estudiada de parejas casadas y que viven juntas.

Palabras clave: satisfacción con la relación, psicometría, matrimonio, unión libre, México.

\begin{abstract}
The study of intimate partner relationships is particularly important because this union is the foundation of the family. Satisfaction with the relationship can be defined as the overall attitude to the relationship and the partner. The Hendrick's Relationship Assessment Scale (RAS) is a instrument commonly used to assess the construct. Previous research papers have showed that this scale has high internal consistency and a single-factor structure. Although there are validation studies of the RAS, these studies used inappropriate statistical techniques to analyze its Likert-type items, and to determine the number of factors; likewise, its factor invariance across sex has not been previously contrasted. Therefore, this study posed the following research questions: Does the RAS have consistent and discriminating items? Basing the analysis on a polychoric correlation matrix, what is its level of internal consistency? How many factors emerge using rigorous empirical methods? Is the single-factor model invariant across sex? In order to answer these research questions, we used a random route probability sampling in this instrument validation study of the RAS. The sample was extracted from the population of married couples or the ones living in consensual union in Monterrey, Mexico. There were 431 female and 376 male participants in the study. The RAS' items were consistent and discriminative. The internal consistency of the scale was excellent in the whole sample (ordinal $\alpha=.93$ ), as well as
\end{abstract}


among female (ordinal $\alpha=.94$ ) and male participants (ordinal $\alpha=.92$ ). Horn's parallel analysis and Velicer's minimum average partial test suggested a one factor solution. Moreover, the single-factor model (with one correlation between the residuals of the two negatively worded items) had a close fit to the data, and its properties of invariance across sex were very acceptable by the Unweighted Least Squares method. We conclude that the scale shows internal consistency and cross-sex validity. We recommend using the scale in the studied population of married couples or the ones living in consensual union.

Keywords: Relationship satisfaction, psychometrics, marriage, consensual union, Mexico.

\section{Introducción}

Dentro de la psicología, el estudio de las relaciones de pareja cobra especial importancia, ya que esta unión constituye la base de la familia y por ende los cimientos para la crianza de los hijos (Anderson, Van Ryzin, \& Doherty, 2010). La satisfacción con la relación de pareja predice la estabilidad de la unión de los padres (Armenta, Sánchez-Aragón, \& Díaz Loving, 2014; Hirschberger, Srivastava, Marsh, Cowan, \& Cowan, 2009); y esta estabilidad tiene una gran importancia no sólo para la felicidad de sus dos miembros (Díaz-Loving \& Rivera-Aragón, 2002), sino también por ser uno de los requisitos necesarios para vivir en una familia funcional que proporcione estructura a su descendencia (Mendoza, Soler, Sainz, Gil, Mendoza, \& Pérez, 2006).

¿Qué se entiende por satisfacción con la relación? Se puede definir como la actitud o juicio valorativo hacia la relación y la pareja (Becerra, Roldán, \& Flores, 2012), o como la valoración del grado de felicidad con la relación y la pareja (Anderson et al., 2010). Armenta et al. (2014) conciben a la satisfacción con la relación como el producto derivado del grado de cercanía y amor en la interacción cotidiana. 
Una persona satisfecha con su relación se siente a gusto con la misma y piensa que su relación y su pareja tienen cualidades positivas. Se postula que la satisfacción existe en un continuo que varía de altamente satisfecho a altamente insatisfecho. Las personas con una alta insatisfacción viven su relación como angustiante, suelen pensar en su abandono o ruptura, lo que amenaza la supervivencia de la misma (Lawrence, Barry, Langer, \& Brock, 2009).

Al hablarse de relación de pareja se incluyen varias tipos de enlaces: marital (relación amorosa entre dos personas bajo un contrato civil y/o religioso que exige la consumación sexual, la convivencia, la fidelidad mutua y el reconocimiento de los hijos que se engendren), unión libre (relación amorosa entre dos personas que viven juntas, ya sea que tengan o no un registro civil de esta situación) y noviazgo (relación amorosa entre dos personas que no viven juntas). Las situaciones de parejas concurrentes (infidelidad) no son abarcadas por este concepto, pero la existencia de una o más parejas concurrentes no constituye un criterio de exclusión para calificar a una persona en una de las tres categorías (matrimonio, unión libre o noviazgo). A su vez estos tres tipos de enlaces de pareja pueden ser entre dos personas de distinto sexo (heterosexuales) o del mismo sexo (homosexuales).

En el presente trabajo, la satisfacción se conceptualiza como una actitud o juicio valorativo global hacia la relación y la pareja (Hendrick, 1988). La investigación se centra en relaciones heterosexuales de matrimonio y unión libre, siendo su objetivo el estudio de las propiedades de consistencia interna y estructura factorial de la una escala que evalúa este concepto como un factor único. Para fundamentar la unidimensionalidad de la satisfacción con la relación de pareja es necesario delimitar el concepto y aclarar la teoría subyacente. Con unidimensionalidad se hace referencia a la hipótesis de que los distintos indicadores de satisfacción con la relación de pareja comparten varianza (covarían), y a esta varianza compartida subyace un único factor. 


\section{La satisfacción con la relación}

Debe señalarse que, en la literatura sobre las relaciones de pareja, el concepto de satisfacción con la relación con frecuencia se confunde con los de felicidad, ajuste y calidad, aunque sí es posible hacer delimitaciones conceptuales. El concepto de felicidad se suele considerar sinónimo al de satisfacción (Anderson et al., 2010; Kamp, Taylor, \& Kroeger, 2008); cuando los conceptos de ajuste y calidad se consideran afines al de satisfacción, pero más amplios (Gottman \& Notarius, 2002). En el contexto de las relaciones de pareja, el concepto de felicidad constituye un juicio valorativo global de la relación, basado en los propios sentimientos (Kamp et al. 2008). El concepto de ajuste diádico influye procesos de comunicación, cohesión, resolución de conflictos y hábitos de convivencia (South, Krueger, \& Iacono, 2009). El concepto de calidad abarca las estrategias de manejo de conflictos, apoyo de la pareja, frecuencia y satisfacción con las relaciones sexuales e intimidad emocional (Chiorri, Day, \& Malmberg, 2014), y se puede entender como la conjunción de los conceptos de satisfacción y manejo del conflicto en la relación (Custer, 2009). No obstante, todos estos conceptos están altamente correlacionados y muestran una capacidad predictiva similar sobre la estabilidad de la pareja y variables de salud de sus miembros (Gottman \& Notarius, 2002).

Es importante remarcar que la satisfacción se trata de un concepto cognitivo y no de un concepto sobre la dinámica de interacción de la pareja. Implica procesos de comparación y juicios valorativos sobre la pareja y la relación presente, siendo la teoría del intercambio social una de las primeras teorías usadas en su conceptualización y que está actualmente vigente (Li \& Fung, 2011).

La satisfacción con la relación tiene diversos determinantes relacionados con la pareja y su contexto. Con respecto a la pareja, el apoyo, el reparto del poder y la comunicación abierta y afectuosa favorecen la satisfacción (Díaz-Loving \& Rivera-Aragón, 
2002; Flores, 2011); por el contrario, rasgos de personalidad, como el neuroticismo y la búsqueda de sensaciones, o estados emocionales, como la depresión, la dificultan (Dávila, Karney, Hall, \& Bradbury, 2003). Con respecto al ambiente, mayor número de hijos, más años de relación y dificultades económicas perjudican a la satisfacción con la relación, sobre todo si el apoyo y la comunicación con la pareja fallan (Stone \& Shackelford, 2007).

La violencia deteriora la satisfacción y puede conducir a un patrón rápido de ruptura si la violencia es recíproca y en escalada. Aunque no haya violencia, la insatisfacción conduce a un patrón lento de ruptura. Por el contrario, si se logra mantener la satisfacción a largo plazo, es esperable la estabilidad de la pareja (Lavner \& Bradbury, 2010). Además del efecto sobre la estabilidad de la pareja, la insatisfacción con la relación actúa como uno de los determinantes de concurrencia de parejas, teniendo un tamaño de efecto mayor sobre la infidelidad femenina que sobre la masculina (Shackelford, Besser, \& Goetz, 2008).

A pesar de que los determinantes de la satisfacción señalados operan con distinto impacto en mujeres y hombres, afectando más el número de hijos y el neuroticismo de la pareja femenina a los hombres y la falta de apoyo y la depresión de la pareja masculina a las mujeres (Carlson, Barden, Daire, \& Greene, 2014; Dávila et al., 2003), la satisfacción promedio reportada es equivalente entre ambos sexos en estudios de población general con parejas casadas (Jackson, Miller, Oka, \& Henry, 2014).

\section{La teoría del intercambio social en la satisfacción con la relación}

Frente a una base empírica de las conceptualizaciones del ajuste diádico y la calidad de la relación, basadas en análisis factoriales, el concepto cognitivo de satisfacción con la relación tiene como base la teoría de la equidad y el intercambio social (Li \& Fung, 2011). 
La satisfacción es la reacción o estado emocional que resulta de un juicio valorativo positivo. La persona está satisfecha con un servicio si se han cumplido sus expectativas o necesidades (Martínez, Peiró, \& Ramos, 2001). No obstante, en el ámbito de relación de pareja, este juicio no se reduce a un cumplimiento de expectativas o necesidades, sino que se amplía a una cuestión de balance y reciprocidad. La teoría del intercambio social postula que los individuos que interactúan evalúan sus relaciones en términos de coste/beneficio o dar/recibir; en la medida en que valoran que existe equilibrio se experimenta satisfacción, y en la medida en que el beneficio equitativo es grande con expectativa de estabilidad o prosperidad se experimenta felicidad (Li \& Fung, 2011). Estos juicios son globales y pueden ser representados como puntos variables en una dimensión bipolar, ubicándose en el extremo izquierdo la insatisfacción y en el extremo derecho la satisfacción (Hendrick, 1988).

\section{Aspectos o dimensiones de la satisfacción con la relación}

Consecuentemente, la evaluación de los conceptos de felicidad y satisfacción con la relación desde la teoría del beneficio equitativo se realizan por medio de escalas de autorreporte unidimensionales (Snyder, Heyman, \& Haines, 2009). En contraste, los conceptos de ajuste y calidad son evaluados por medio de inventarios multidimensionales, que suelen incluir un factor de satisfacción y tienen una base empírica derivada de estudios de pareja y de análisis factoriales (Snyder et al., 2009).

Debe señalarse que hay evaluaciones multidimensionales de satisfacción, que separan la satisfacción con la pareja de varios aspectos de la relación, pero que conceptualmente podría ser más bien consideradas evaluaciones de conceptos afines, pero más amplios, como el de calidad o el de ajuste diádico (Lawrence et al., 2009). 


\section{Satisfacción con la relación marital y su medición}

El concepto de satisfacción marital se refiere a un sentimiento de felicidad general con la relación marital y el cónyuge. Precedió al concepto de satisfacción con la relación, siendo este último un intento de ampliar el primero a distintos tipos de relación de pareja, como noviazgo, cohabitación y matrimonio (Sabatelli, 2009). En los ochenta la evaluación de la satisfacción marital partió de medidas unidimensionales. A mediados de los noventa evolucionó hacia medidas multidimensionales, lo que contribuyó a la confusión conceptual presente (Lawrence et al., 2009).

Uno de los primeros instrumentos de medida desarrollados para evaluar satisfacción con la relación marital fue la Escala de Satisfacción Marital de Kansas (Kansas Marital Satisfaction Scale [KMS]; Schumm, Nichols, Schectman, \& Grinsby, 1983). Se trata de una escala breve de tres ítems tipo Likert que se responden en una escala ordinal con un rango de siete valores. En un estudio de meta-análisis, Graham, Diebels y Barnow (2011) estimaron una consistencia interna aceptable entre los tres ítems de la KMS ( $\alpha$ de Cronbach $=.79$, IC 95\%: .77, .80), aunque observaron heterogeneidad significativa entre las 122 investigaciones seleccionadas para realizar la estimación ( $Q$ de Cochrane $=2,479.1, p<$ $\left..001 ; I^{2}=96\right)$. Schumm et al. (1986) reportaron que la estructura factorial de los tres ítems de la KMS es unidimensional. La KMS es una escala ampliamente usada en el ámbito clínico y se haya baremada y validada usando como criterio la Escala de Ajuste Diádico (Crane, Middleton, \& Bean, 2000; Ward, Lundberg, Zabriskie, \& Berrett, 2009).

Otro de los primeros instrumentos de medida fue el Índice de Calidad del Matrimonio (Quality of Marriage Index [QMI]; Norton, 1983). El QMI evalúa en qué grado la persona se siente feliz con su matrimonio, por lo que se considera que es una escala de 
satisfacción con la relación marital. El QMI está integrado por cinco ítems tipo Likert que se responden en una escala ordinal con un rango de siete valores. En el estudio de un metaanálisis, Graham et al. (2011) reportaron una consistencia interna excelente entre los cinco ítems del QMI ( $\alpha$ de Cronbach $=.94$, IC 95\%: .94, .95), aunque se observó heterogeneidad entre 189 investigaciones seleccionadas para realizar la estimación $(Q$ de Cochrane $=$ $\left.3,707.8, p<.001 ; I^{2}=95\right)$. Norton (1983) reportó que la estructura de los cinco ítems es unidimensional.

Otro instrumento de evaluación es el Índice de Satisfacción Marital (Hudson’s Index of Marital Satisfaction [IMS]; Hudson, 1992) que está compuesto por 25 ítems tipo Likert que se responden en una escala ordinal con un rango de cinco valores. En un estudio de validación realizado en España, con una muestra de 183 mujeres, Iraurgi, Sanz y Martínez (2009) obtuvieron una consistencia interna excelente entre los 25 ítems del IMS ( $\alpha$ de Cronbach $=.97)$. Los investigadores españoles señalaron que el IMS se puede considerar una escala unidimensional, al presentar dos factores con correlación alta y definidos por los ítems directos e inversos, siendo el ajuste del modelo unidimensional con 25 indicadores por Máxima Verosimilitud de bueno $\left(\chi^{2}[275]=307.78, p=.547 ; \chi 2 / g l=\right.$ 1.12) a aceptable $(G F I=.88, C F I=.89$ y $R M S E A=.07)$.

Como ya se ha señalado, existen medidas multidimensionales de la satisfacción marital, pero estos inventarios deben considerarse evaluaciones de ajuste y calidad (Lawrence et al., 2009), como las Escalas de Temas de Enriquecimiento y Cuidado de las Relaciones, Comunicación y Felicidad (Evaluation and Nurturing Relationship Issues, Communication, and Happiness [ENRICH] scales; Olson, Fournier, \& Druckman, 1985), la 
Escala de Satisfacción Marital (ESM; Pick \& Andrade, 1988), o el Inventario Multifacético de la Satisfacción Marital (IMSM; Cañetas, Rivera-Aragón, \& Díaz-Loving, 2000).

\section{Medición de la satisfacción con la relación de pareja}

Aunque hay instrumentos que específicamente apelan a la relación marital, en la práctica se aplican tanto a situaciones de matrimonio como en unión libre, incluso a situaciones de noviazgo, dejando abierta la interpretación de los términos "mi pareja" y "mi relación" y evitando los términos "cónyuge" y "matrimonio".

Entre los instrumentos que evalúan de forma específica la satisfacción con la relación de pareja sobresale la Escala de Valoración de la Relación (Relationship Assessment Scale [RAS]; Hendrick, 1988). Se trata del instrumento más ampliamente usado y estudiado en la evaluación de parejas, ya sea de parejas con distinto estado civil (casadas, en unión libre o noviazgo) o con distinta orientación sexual (Graham et al., 2011).

La RAS es una medición unidimensional que se basa en la teoría del intercambio social (Hendrick, Dicke, \& Hendrick, 1998). Está integrada por siete ítems tipo Likert que se responden en una escala ordinal con un rango de cinco valores. En un estudio de metaanálisis, tras seleccionar 196 investigaciones publicadas entre 1988 y 2010, Graham et al. (2011) estimaron una consistencia buena entre los siete ítems de la RAS ( $\alpha$ de Cronbach $=$ .87, IC 95\%: .86, .88). En esta estimación de la consistencia interna no se encontró sesgo de publicación o tendencia a publicar sólo estudios con resultados positivos $(N$ de Orwin relativamente alta $=285)$; no obstante, sí se observó heterogeneidad entre los estudios seleccionados, como muestra la combinación de un índice de estabilidad alto $\left(I^{2}=94\right)$ y la prueba $Q$ de Cochrane estadísticamente significativa $(Q=3,007.4, p<.001)$. Al estimar correlaciones bivariadas entre la alfa reportada y las características sociodemográficas de 
las muestras de cada estudio, hallaron correlaciones significativas y bajas con el porcentaje de personas con estudios universitarios $(r[190]=.27, p<.001)$, con el porcentaje de personas de otra nacionalidad $(r[196]=.21, p<.01)$ y con el sexo $(r[187]=-.18, p<.05)$; y correlaciones significativas y moderadas con la edad $(r[149]=.42, p<.001)$, con el estar casado o no $(r[122]=.36, p<.001)$ y con la duración de la relación $(r[108]=.35, p<$ .001 ), lo que explica parte de la heterogeneidad significativa entre los 196 estudios seleccionados (Graham et al., 2011).

Hendrick (1988), en una muestra de 125 estudiantes universitarios estadounidenses con relaciones de noviazgo, observó que el número de factores de los siete ítems fue uno por el criterio de Kaiser (número de autovalores de la matriz de correlaciones mayores que uno). Al extraer el componente se explicó el $46 \%$ de la varianza total y las cargas factoriales variaron de .57 a .76. En una segunda muestra de 57 parejas de novios, replicó la estructura de un factor, siendo $57 \%$ la varianza total explicada; obtuvo una consistencia interna buena $(\alpha$ de Cronbach $=.86$ ); y validez convergente, al ser la correlación entre la RAS y la DAS muy alta $(r=.80, p<.001)$.

La RAS se halla adaptada y validada en México (Moral, 2008; Oropeza, Armenta, García, Padilla y Díaz-Loving, 2010; Sáenz, 2014). Moral (2008) hizo una traducción de la RAS con adaptaciones a la población de parejas casadas que posteriormente fue usada por Sáenz (2014), y Oropeza et al. (2010) realizaron otra traducción más literal de la escala original.

Moral (2008), en una muestra no probabilística de 100 parejas heterosexuales casadas que residían en la ciudad de Monterrey del estado de Nuevo León $(n=200)$, exploró la estructura factorial de los siete ítems de la RAS. El número de factores fue uno 
por el criterio de Kaiser. Tras la extracción del factor por Ejes Principales se explicó el 38.5\% de la varianza total. Por Máxima Verosimilitud, el ajuste del modelo unidimensional con siete indicadores fue aceptable: $\chi 2 / g l=3.08, G F I=.92, A G F I=.98, C F I=.98 \mathrm{y}$ RMSEA $=.07$. La consistencia interna de los siete ítems fue buena $(\alpha$ de Cronbach $=.81)$. En este estudio, la escala RAS no sólo mostró consistencia interna y validez estructural, sino también validez convergente al tener una correlación muy alta $(r[200]=.81, p<.001)$ con la ESM de Pick y Andrade (1988).

Oropeza et al. (2010), en una muestra no probabilística de 204 personas que vivían con una pareja heterosexual en unión libre en la ciudad de México, hallaron una consistencia interna buena $(\alpha$ de Cronbach $=.88)$ entre los cinco ítems directos de la RAS (ítems 1, 2, 3, 5 y 6), los cuales fueron seleccionados tras descartar los dos ítems inversos por no ser discriminativos con un nivel de significación de .01: ítem $4(t[100]=2.43, p=$ $.017>.01)$ e ítem $7(t[100]=1.76, p=.082>.01)$. Por análisis de Componentes Principales, los cinco ítems seleccionados definieron un componente con cargas factoriales altas que variaron de .56 a .85 con una media de .71 .

Sáenz (2014), en una muestra no probabilística de 305 parejas heterosexuales casadas o en unión libre reclutada en la ciudad de Monterrey $(n=610)$, estudió las propiedades psicométricas de la RAS. Obtuvo una consistencia interna buena en la muestra de mujeres $(\alpha$ de Cronbach $=.81)$, y aceptable en la muestra conjunta $(\alpha$ de Cronbach $=$ .78) y en la de hombres $(\alpha$ de Cronbach $=.75)$. El número de factores por el criterio de Kaiser fue dos. Al ser extraídos por Componentes Principales con una rotación de la matriz de componentes por el método Varimax, el primer componente quedó definido por los cinco ítems directos y el segundo por los dos ítems inversos (ítems 4 y 7). Ambos 
componentes explicaron el $53.7 \%$ de la varianza total. La configuración de una estructura de dos factores correlacionados, en la cual un factor con más consistencia y mayor media agrupa a los ítems directos y otro factor con menor consistencia y menor media agrupa a los ítems inversos, se atribuye a un efecto metodológico en relación con la dificultad para comprender los ítems redactados en sentido contrario al rasgo evaluado y no una diferencia con base teórica, por lo que la estructura se puede interpretar a nivel teórico como unidimensional (Iraurgi et al., 2009; Lindwall et al., 2012; Sauro \& Lewis, 2011; Wu, 2008), como sería el caso del estudio de Sáenz (2014).

En un estudio piloto hecho en Suecia, Rask et al. (2010) reportaron una estructura de dos factores correlacionados no atribuible a la dificultad comprensiva de los ítems inversos. Un factor se denominó relación construida sobre las expectativas y la satisfacción de necesidades o amor instrumental (ítems 1, 2, 3 y 5) y el otro factor se denominó relación construida sobre el amor y la devoción o amor romántico (ítems 4, 6 y 7).

En población general, la RAS ha revelado que el reporte de la satisfacción con la relación se sesga hacia el polo de las puntuaciones altas, correspondiendo el promedio a una respuesta de satisfacción sin diferencia estadísticamente significativa entre ambos sexos (Dinkel \& Balck, 2005; Hendrick et al., 1998; Moral, 2008; Sáenz, 2014). En población clínica, se sesga hacia el polo de la insatisfacción con un promedio significativamente menor en mujeres que en hombres (Jackson et al., 2014). No obstante, en mujeres mexicanas que sufren violencia de pareja y permanecen en la relación disfuncional, Canales (2011) observó que se reporta satisfacción con la relación. 


\section{Métodos para estudiar las propiedades psicométricas de escalas con ítems ordinales}

En las últimas décadas ha habido avances importantes en el análisis de escalas integradas por ítems con una escala de medida ordinal (Basto \& Pereira, 2012). Se ha señalado que el coeficiente de correlación producto-momento de Pearson, ideado para dos variables numéricas continuas con distribución normal, subestima la asociación lineal entre dos variables ordinales. En su lugar se recomienda el uso de la correlación policórica que es una estimación indirecta de la correlación a partir de las dos variables continuas subyacentes a la contingencia o cruce de los datos ordinales. Consecuentemente se recomienda extraer los factores de la matriz de correlaciones policóricas en lugar de la matriz de correlaciones de Pearson (Basto \& Pereira, 2012; Garrido, Abad, \& Ponsoda, 2013; Hoffmann, Stover, De la Iglesia, \& Fernández, 2013; Holgado, Chacón, Barbero, \& Vila, 2008; Olsson, 1979; Pearson, 1900); no obstante, se previene de las dificultades de cálculo, la necesidad de estabilidad en la estimación y lo restrictivo de los supuestos de la correlación policórica, como el de normalidad bivariada (Choi, Peters, \& Mueller, 2010; Ekström, 2009; Ferrando \& Lorenzo-Seva, 2014; Yule, 1912).

Se ha remarcado la importancia de determinar el número de factores en el análisis factorial exploratorio, usando la convergencia de métodos empíricos matemáticamente precisos, como el de análisis paralelo de Horn (número de autovalores de la curva de sedimentación de los datos observados que quedan por encima del punto de intersección entre dicha curva y la correspondiente al percentil 50 o 95 entre las curvas generadas con datos al azar con el mismo número de casos y variables que el de la muestra observada), el promedio mínimo de las correlaciones parciales al cuadrado de Velicer (número de componentes parcializados al alcanzarse este promedio mínimo) y el análisis de 
coordenadas óptimas de Raiche, Roipel y Blais (número de autovalores con gradientes asociados decrecientes que quedan por encima del punto de intersección del análisis paralelo de Horn), y se ha criticado al criterio de Kaiser como el más inadecuado para este fin (Courtney, 2013).

Se ha indicado que los métodos apropiados para explorar y contrastar modelos estructurales con variables ordinales son Mínimos Cuadrados No Ponderados y Mínimos Cuadrados Ponderados, lo cuales parten de la matriz de correlaciones reducida (con estimaciones de la comunalidad en la diagonal principal). Se señala que análisis de Componentes Principales, que parte de la matriz de correlaciones (con unos en la diagonal principal), no es un método de análisis factorial exploratorio, sino que se trata de un método de reducción de variables (Costello \& Osborne, 2005; Lloret, Ferreres, Hernández, \& Tomás, 2014; Valdivieso, 2013).

También se ha señalado que el coeficiente $\alpha$ de Cronbach subestima la consistencia interna entre variables ordinales, al estar basado en la matriz de correlaciones de Pearson. En su lugar se recomienda el empleo del coeficiente alfa ordinal que se basa en la matriz de correlaciones policóricas (Elosua \& Zumbo, 2008).

Aunque existen estudios previos de validación de la RAS en México y otros países, estos han empleado técnicas de análisis inadecuadas para la escala ordinal de sus siete ítems. La mayoría de los estudios informan de un factor, pero también se han reportado soluciones bifactoriales en México (Sáenz, 2014) y en Suecia (Rask et al., 2010). Precisamente, una de las limitaciones de los estudios previos es que el número de factores de la RAS se ha determinado por el criterio de Kaiser y no por la convergencia de los criterios matemáticos empíricos más estrictos, como el de Horn, Velicer y coordenadas 
óptimas. Aunque debe señalarse que, en un estudio publicado en Brasil, se consideró la convergencia de los criterios de Kaiser, Cattell y Horn, y la convergencia fue en un factor (Cassepp \& Pasquali, 2011).

Tampoco se ha contrastado la invarianza del modelo de un factor entre ambos sexos, asumiéndose que es válido para hombres y mujeres, al considerarse que los sistemas de representación social y valores culturales sobre la relación de pareja son semejantes entre ambos sexos (Armenta et al., 2014; López \& Rodríguez, 2008), y que esta invarianza ya ha sido demostrada en conceptos afines, como el de ajuste diádico (South et al., 2009) y el de engrandecimiento marital o tendencia a exagerar las cualidades positivas y aminorar las cualidades negativas del cónyuge y la relación marital (O'Rourke \& Cappeliez, 2001).

Cabe preguntarse si: ¿se aplican los métodos correctos para estudiar las propiedades de consistencia interna y estructura factorial, se reproducirán los resultados hasta ahora reportados?, ¿la estructura unifactorial es invariante entre ambos sexos?

\section{Objetivos e hipótesis}

Graham et al. (2011) indicaron que la RAS es un instrumento de medida con una consistencia interna buena, pero afectada en su estimación por las características sociodemográficas de la muestra. A consecuencia de esta limitación, se recomienda usar muestras de tamaño grande y procedimientos de muestreo probabilísticos en el diseño de los estudios, y limitar las conclusiones a la población de la cual se extraiga la muestra. Al seguir esta sugerencia, se contrarresta la tendencia a usar muestras no probabilísticas de tamaño limitado y a sobregeneralizar los resultados (Breakwell, Smith, \& Wright, 2012).

Considerando la escala de medida ordinal de los siete ítems que integran la RAS, la presente investigación en la población de parejas casadas y en unión libre de la ciudad de 
Monterrey en México tiene como objetivos: 1) describir las distribuciones de los siete ítems (por los cuartiles, el rango semintercuartílico, el coeficiente de asimetría intercuartilíco y el coeficiente de curtosis percentilíco corregido), estudiar sus propiedades de discriminación (por la prueba U de Mann-Whitney) y de consistencia interna (por el coeficiente de correlación poliserial y el coeficiente alfa ordinal de Elosua y Zumbo (2008), excluyendo el ítem en el cálculo de ambos coeficientes); 2) estimar la consistencia interna entre los siete ítems (por el coeficiente alfa ordinal); 3) determinar el número de factores por la convergencia de los criterios empíricos de Horn, Velicer y coordenadas óptimas; y 4) contrastar el modelo de un factor por mínimos cuadrados sin ponderar desde la matriz de correlaciones policóricas; asimismo, comprobar su invarianza entre ambos sexos, especificando cuatro modelos anidados en restricciones acumulativas entre los parámetros de ambas muestras, para lo cual se parte del modelo de un factor con sus parámetros libres en cada muestra, a continuación se impone la igualdad entre los pesos de medida de mujeres y hombres, en la siguiente restricción se añade la igualdad entre la varianza del factor en cada sexo y por último se añade la igualdad entre los residuos de medida de cada muestra; y 5) explorar y contrastar modelos alternos al de un factor, en caso de no ser sustentado por los tres criterios empíricos para determinar el número de factores, mostrar mal ajuste a los datos y presentar variación estructural entre ambos sexos.

Desde los resultados de Hendrick (1988), Hendrick et al. (1998), Moral (2008), Oropeza et al. (2010), Cassepp y Pasquali (2011), Graham et al. (2011) y Sáenz (2014), se espera que: 1) los siete ítems sean consistentes y discriminativos, siendo los dos ítems inversos (ítems 4 y 7) los más débiles en consistencia interna y discriminación; 2) una consistencia interna entre los siete ítems al menos buena $(\alpha$ ordinal $\geq .80)$ o incluso 
excelente ( $\alpha$ ordinal $\geq 90$ ), en concordancia con la unidimensionalidad subyacente (Tavakol \& Dennick, 2011); 3) convergencia de los tres criterios empíricos para determinar el número de factores (Horn, Velicer y coordenadas óptimas) en un factor; 4) un ajuste a los datos del modelo de un factor entre aceptable y bueno, aunque la correlación entre los residuos de medida de los ítems 4 y 7 pudiese mejorar el ajuste; asimismo, unas propiedades de invarianza entre ambos sexos aceptables o buenas, especialmente en los modelos sin restricciones y con restricciones en los pesos de medida; y 5) como modelo alterno al de un factor se podría definir un modelo de dos factores correlacionados, un factor configurado por los cinco ítems directos y otro por los dos ítems inversos, como en el estudio de Sáenz (2014); este modelo bifactorial, por el número el número insuficiente de indicadores del segundo factor y por no poseer una sustantividad teórica en su interpretación, se contemplaría como una corrección al modelo de un factor, especificando una correlación entre los residuos de los dos ítems inversos; otra alternativa sería el modelo de dos factores correlacionados de Rask et al. (2010), con un factor de amor instrumental (ítems 1, 2, 3 y 5) y el otro factor de amor romántico (ítems 4, 6 y 7).

\section{Alcances del estudio}

Este estudio introduce una nueva forma de validar escalas tipo Likert con métodos adecuados para ítems con una escala de medida ordinal, haciendo uso de programas al alcance de cualquier investigador en psicología, lo cual es novedoso no sólo en América Latina, sino en el resto de las regiones continentales. La investigación se centra en una variable muy importante dentro de las problemáticas de pareja, ya sea violencia, ruptura o salud, y se enfoca hacia las propiedades psicométricas de una escala muy útil y usada en la investigación y la terapia de pareja, como es la RAS. Se usa una traducción al español muy 
apegada a la escala original. Además, se emplea una muestra probabilística, lo que le da un valor agregado sobre la mayoría de los estudios publicados sobre las propiedades psicométricas de la RAS que han usado métodos no probabilísticos de muestreo.

\section{Método}

\section{Diseño}

La presente investigación es un estudio instrumental. En esta categoría se incluyen a los estudios enfocados al desarrollo de pruebas, incluyendo su diseño y adaptación (Montero \& León, 2002). Al estudiarse las propiedades de consistencia interna, estructura factorial e invarianza entre sexos se usó un diseño ex post facto transversal, al no requerirse manipulación de variables ni seguimiento de casos (Breakwell et al., 2012).

\section{Participantes}

Los criterios de inclusión fueron: tener al menos 18 años, estar casado o vivir en unión libre con una pareja del sexo opuesto, residir en la ciudad de Monterrey en México, saber leer y escribir y prestar el consentimiento informado. Los criterios de eliminación fueron: cuestionario incompleto e informar que la pareja estuvo presente al momento de ser contestado, leyendo o preguntando por las respuestas.

Se empleó un muestreo probabilístico usando el método de rutas aleatorias. Este tipo de muestreo busca asegurar una cobertura representativa de un área geográfica ante la falta de censo. Establecida el área, se definen uno o más puntos de partida, desde los que se trazan rutas en las que se van seleccionando los participantes (Breakwell et al., 2012).

Se contactó con 1,000 personas (500 mujeres y 500 hombres) que cumplían criterios de inclusión. E1 17.8\% de las personas contactadas (61 de las 500 mujeres [12.2\%] y 117 de los 500 hombres [23.4\%]) se rehusaron a participar, siendo la diferencia de consentimiento 
concedido para participar estadísticamente significativa entre ambos $\operatorname{sexos}\left(\chi^{2}[1, N=1,000]\right.$ $=21.43, p<.001 ;$ con la corrección con continuidad de Yates: $\chi^{2}[1, N=1,000]=20.67, p<$ .001). Los motivos expresados fueron falta de tiempo o estar muy ocupado (61\%), desinterés en participar en encuestas (19\%), desconfianza en dar datos personales (12\%), que esté presente la familia o la pareja (7\%) y otros motivos (1\%), sin diferencia estadísticamente significativa entre ambos $\operatorname{sexos}\left(\chi^{2}[4, N=178]=8.86, p=.065\right)$. En 15 casos (siete hombres y ocho mujeres) se aplicaron criterios de eliminación, en 12 por presencia del cónyuge (siete mujeres y cinco hombres) y en tres por datos incompletos (una mujer y dos hombres).

La muestra analizada con todos sus datos completos fue de 807 participantes, de los cuales $431(53.4 \%)$ fueron mujeres y $376(46.6 \%)$ hombres, sin diferencia de frecuencia estadísticamente significativa entre ambos sexos por la prueba binomial $(p=.057)$. Con respecto al estado civil, 727 (90\%) indicaron estar casados y 80 (10\%) en unión libre, sin diferencia de frecuencia estadísticamente significativa entre ambos $\operatorname{sexos}\left(\chi^{2}[1, N=807]=\right.$ 1.02, $p=.313$, con la corrección por continuidad de Yates: $\chi^{2}[1, N=807]=0.79, p=.373$ ). La media de años de vivir juntos fue $11.61(D E=9.33)$, variando de 6 meses a 43 años, sin diferencia de medias estadísticamente significativa entre ambos sexos $(t[805]=0.23, p=$ .821). De las 807 personas encuestadas, 677 (84\%) dijeron tener hijos y 130 (16\%) no tener hijos. La media de hijos fue $1.99(D E=1.30)$, variando de 0 a 9 , sin diferencia de medias estadísticamente significativa entre ambos sexos $(t[805]=0.96, p=.339)$ (véase Tabla 1 ). 
Tabla 1

Frecuencia y porcentajes de las variables sociodemográficas

\begin{tabular}{|c|c|c|c|c|}
\hline Variables & Valores & Hombres & Mujeres & Total \\
\hline \multirow[t]{3}{*}{ Sexo } & & $376(46.6 \%)$ & $431(53.4 \%)$ & $807(100 \%)$ \\
\hline & {$[18,29]$} & $126(33.5 \%)$ & $159(36.9 \%)$ & $285(35.3 \%)$ \\
\hline & {$[30,39]$} & $103(27.4 \%)$ & $127(29.5 \%)$ & $230(28.5 \%)$ \\
\hline Años & {$[40,49]$} & $104(27.7 \%)$ & $116(26.9 \%)$ & $220(27.3 \%)$ \\
\hline \multirow{4}{*}{ de edad } & {$[50,59]$} & $34(9 \%)$ & $26(6.0 \%)$ & $60(7.4 \%)$ \\
\hline & {$[60,94]$} & $9(2.4 \%)$ & $3(0.7 \%)$ & $12(1.5 \%)$ \\
\hline & Primaria & $80(21.3 \%)$ & $93(21.6 \%)$ & $173(21.4 \%)$ \\
\hline & Secundaria & $92(24.5 \%)$ & $139(32.3 \%)$ & $231(28.6 \%)$ \\
\hline \multirow[t]{4}{*}{ Escolaridad } & Media superior & $108(28.7 \%)$ & $110(25.5 \%)$ & $218(27 \%)$ \\
\hline & Licenciatura & $84(22.3 \%)$ & $80(18.6 \%)$ & $164(20.3 \%)$ \\
\hline & Posgrado & $12(3.2 \%)$ & $9(2.1 \%)$ & $21(2.6 \%)$ \\
\hline & Sí & $343(91.2 \%)$ & $384(89.1 \%)$ & $727(90.1 \%)$ \\
\hline \multirow{2}{*}{ Casado } & No & $33(8.8 \%)$ & $47(10.9 \%)$ & $80(9.9 \%)$ \\
\hline & {$[0,7.16]$} & $169(44.9 \%)$ & $192(44.5 \%)$ & $361(44.7 \%)$ \\
\hline Años & {$[7.17,14.33]$} & $69(18.4 \%)$ & $77(17.9 \%)$ & $146(18.1 \%)$ \\
\hline de & {$[14.34,21.50]$} & $74(19.7 \%)$ & $97(22.5 \%)$ & $171(21.2 \%)$ \\
\hline matrimonio & {$[21.51,28.66]$} & $44(11.7 \%)$ & $47(10.9 \%)$ & $91(11.3 \%)$ \\
\hline \multirow[t]{2}{*}{ o unión libre } & {$[28.67,35.83]$} & $13(3.5 \%)$ & $14(3.2 \%)$ & $27(3.3 \%)$ \\
\hline & {$[35.84,43]$} & $7(1.9 \%)$ & $4(0.9 \%)$ & $11(1.4 \%)$ \\
\hline
\end{tabular}




\begin{tabular}{|c|c|c|c|c|}
\hline Variables & Valores & Hombres & Mujeres & Total \\
\hline Ingresos & $<3000$ & $23(6.1 \%)$ & $38(8.8 \%)$ & $61(7.6 \%)$ \\
\hline económicos & {$[3,000,5,999]$} & $96(25.5 \%)$ & $124(28.8 \%)$ & $220(27.3 \%)$ \\
\hline familiares & {$[6,000,11,999]$} & $109(29 \%)$ & $117(27.1 \%)$ & $226(28.0 \%)$ \\
\hline al mes & {$[12,000,23,999]$} & $90(23.9 \%)$ & $84(19.5 \%)$ & $174(21.6 \%)$ \\
\hline en el último & {$[24,000,47,999]$} & $33(8.8 \%)$ & $44(10.2 \%)$ & $77(9.5 \%)$ \\
\hline \multirow[t]{3}{*}{ año en pesos } & {$[48,000,91,999]$} & $16(4.3 \%)$ & $18(4.2 \%)$ & $34(4.2 \%)$ \\
\hline & $\geq 92,000$ & $9(2.4 \%)$ & $6(1.4 \%)$ & $15(1.9 \%)$ \\
\hline & 0 & $56(14.9 \%)$ & $74(17.2 \%)$ & $130(16.1 \%)$ \\
\hline & 1 & $75(19.9 \%)$ & $74(17.2 \%)$ & $149(18.5 \%)$ \\
\hline & 2 & $105(27.9 \%)$ & $132(30.6 \%)$ & $237(29.4 \%)$ \\
\hline & 3 & $96(25.5 \%)$ & $115(26.7 \%)$ & $211(26.1 \%)$ \\
\hline & 4 & $31(8.2 \%)$ & $29(6.7 \%)$ & $60(7.4 \%)$ \\
\hline & $\geq 5$ & $13(3.5 \%)$ & $7(1.6 \%)$ & $20(2.5 \%)$ \\
\hline & Cris. católica & $296(78.7 \%)$ & $366(84.9 \%)$ & $662(82.0 \%)$ \\
\hline Afiliación & Cris. no católica & $39(10.4 \%)$ & $37(8.6 \%)$ & $76(9.4 \%)$ \\
\hline \multirow[t]{2}{*}{ religiosa } & Otra & $5(1.3 \%)$ & $8(1.9 \%)$ & $13(1.6 \%)$ \\
\hline & Ninguna & $36(9.6 \%)$ & $20(4.6 \%)$ & $56(6.9 \%)$ \\
\hline
\end{tabular}

La media de edad fue 35.40 años $(D E=10.41)$ y la mediana 34 , variando de 18 a 94 años. La media de años de edad de las mujeres $(M=34.58$, IC 95\%: $33.84,35.32)$ fue significativamente menor $(t[805]=2.38, p=.018)$ que la de los hombres $(M=36.32$, IC 95\%; 35.41, 37.23). La mediana de escolaridad $(M d n=2)$ correspondió a secundaria (de 7 
a 9 años de escolaridad) y la media $(M=2.51)$ quedó entre estudio de secundaria y media superior (en torno a 10 años de escolaridad). La tendencia central de escolaridad fue significativamente mayor $\left(Z_{U}=-2.61, p=.009\right)$ en hombres (media superior, de 10 a 12 años de escolaridad) que en mujeres (secundaria, de siete a nueve años de escolaridad). La mediana y la media de ingreso mensual de la familia correspondieron al rango de 6,000 a 11,999 pesos, sin diferencia de tendencia central significativa entre ambos sexos $\left(Z_{U}=-\right.$ $1.41, p=.079)$. Con respecto a la religión, 662 de los 807 participantes (82\%) dijeron ser cristianos católicos, $76(9.4 \%)$ ser cristianos no católicos, $13(1.6 \%)$ pertenecer a otras religiones y 56 (6.9\%) no pertenecer a ninguna; con diferencia de frecuencia significativa $\left(\chi^{2}[3, N=807]=9.01, p=.029\right)$, más mujeres señalaron ser cristianas católicas $(85 \%$ versus 79\%) y más hombres no tener religión (10\% versus 5\%) (véase Tabla 1).

En la población de Monterrey se reporta 98 hombres por cada 100 mujeres, una mediana de 30 años de edad que sube a 34 al eliminar al 31\% de la población menor de 18 años, una media de 9.5 años de escolaridad (entre primer semestre y segundo de educación media superior), una media de ingresos mensuales de 6,700 pesos mexicanos, una media de dos hijos, y el 83\% de la población con afiliación religiosa cristiana católica, 7.4\% cristiana no católica, 2.5\% de otra religión y 6.9\% sin religión (INEGI, 2011). Estos datos poblacionales son estadísticamente equivalentes a los muestrales, como en la frecuencia de ambos sexos $\left(\chi^{2}[1, N=807]=1.87, p=.171\right)$ y de la afiliación religiosa $\left(\chi^{2}[3, N=807]=\right.$ 7.13, $p=.068)$ y en la media de hijos $(t[806]=-0.27, p=.787)$, o muy semejantes, como en la mediana de edad (34 años), media de escolaridad (entre secundaria y media superior) y la media de ingresos (rango de 6,000 a 11,999 pesos). 
Entre los adultos de la zona metropolitana de Nuevo León, 49.9\% están casados, $5.7 \%$ en unión libre, $34.6 \%$ solteros, $5 \%$ viudos, $2.8 \%$ separados y $1.9 \%$ divorciados (Secretaría de Salud de Nuevo León, 2013), Si se reduce la población a la de adultos casados y en unión libre, se tendría $89 \%$ casados y $11 \%$ en unión libre, esto es nueve personas casadas por cada persona en unión libre, lo que es un porcentaje equivalente al muestral $\left(\chi^{2}[1, N=807]=0.97, p=.324\right)$.

\section{Instrumento}

Escala de Valoración de la Relación (RAS; Hendrick, 1988). Se usó de la traducción al español de Moral (2008) derivada del método de traducción reversa (véase Figura 1), sin las modificaciones que se introdujeron para hacer que la escala fuese más específica a la relación marital en el estudio publicado en 2008. La escala está integrada por siete ítems tipo Likert con un rango ordinal de respuestas de uno a cinco. Las puntuaciones en la RAS se obtienen por suma simple de los ítems. Dos ítems están redactados en sentido contrario a satisfacción (ítems 4 y 7), por lo que, antes de ser sumados, hay que invertir sus puntuaciones: $1=5,2=4,3=3,4=2$ y $5=1$. El rango de la escala es de 7 a 35 . Una mayor puntuación refleja una mayor satisfacción con la relación de pareja. 
Por favor indique con una $\mathrm{X}$ el número que responde mejor como opción de respuesta $\mathrm{a}$ cada pregunta sobre su relación de pareja. Conteste lo más sinceramente posible

1) ¿De qué manera considera usted que su pareja satisface sus necesidades?

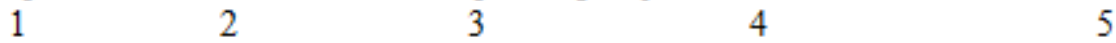

Pobremente Algo Término medio Bastante Extremadamente bien

2) En general, ¿hasta qué punto está satisfecha/o con su relación?

$\begin{array}{lllll}1 & 2 & 3 & 4 & 5\end{array}$

Muy insatisfecho Insatisfecho Término medio Satisfecho Muy satisfecho

3) ¿Qué tan buena es su relación en comparación con la mayoria?

$1 \quad 2 \quad 3 \quad 4$

Muymala Mala Términomedio Buena

4) ¿Con qué frecuencia desea no haberse implicado con su pareja?

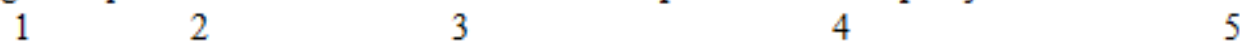

Nunca A veces Frecuentemente Muy frecuentemente

5) ¿Hasta qué punto su relación satisface sus expectativas inicial es?

$\begin{array}{lllll}1 & 2 & 3 & 4 & 5\end{array}$

Nada Algo Término medio Bastante Totalmente

6) ¿Cuánto ama a su pareja?

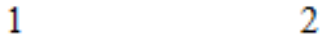

Muy poco

Poco Término medio

4

Mucho

7) ¿Cuántos problemas hay en su relación de pareja?

$\begin{array}{ccccc}1 & 2 & 3 & 4 & 5 \\ \text { Poy pocos } & \text { Pocos } & \text { Lo normal } & \text { Muchos } & \text { Muchisimos }\end{array}$

Figura 1. Escala de Valoración de la Relación (RAS).

\section{Procedimiento}

En el último censo de población y vivienda (Instituto Nacional de Estadística, Geografía e Informática [INEGI], 2010), la población de la ciudad de Monterrey fue de 1 millón 135 mil 550 individuos. La población adulta representa dos tercios de la pirámide poblacional, con lo que 750,000 regiomontanos son adultos (INEGI, 2010). Entre los adultos de Monterrey, 55.7\% están casados o en unión libre, 34.6\% solteros, 5\% viudos, 2.8\% separados y 1.9\% divorciados (Secretaría de Salud de Nuevo León, 2013), con lo que la población objeto de estudio se reduciría a un aproximado de 417,750 adultos. Si se desea estimar una media (escala RAS), bajo un modelo de curva normal, con una desviación 
estándar esperada de 4.49 en un rango de 7 a 35 (Moral, 2008) y con un error absoluto de estimación de 0.31 , se requeriría una muestra con al menos 806 individuos. Se requeriría este mismo tamaño de muestra, si la desviación estándar esperada fuese 0.59 en un rango de 1 a 5 (Sáenz, 2014) y el error absoluto sería 0.04.

De forma aleatoria se seleccionaron 81 direcciones de la ciudad a partir de la Guía Roji (dos direcciones por encuestador). Cada dirección se tomó como punto de partida de un itinerario. En cada itinerario se recolectó 10 unidades (prestando el consentimiento informado y con datos completos). Se pretendía que fueran cinco mujeres y cinco hombres independientes (uno por casa) en cada itinerario.

Se solicitó el consentimiento expreso informado de los participantes, se garantizó el anonimato de sus respuestas, no se solicitó ningún dato de identificación personal y se informó sobre la identidad del responsable del estudio, a quien se podía contactar por correo electrónico para cualquier cuestión suscitada por el cuestionario, conforme con las normas éticas de la American Psychological Association (2002).

El encuestador tocaba el timbre o puerta de la casa. Si esta era abierta, se presentaba el entrevistador como un ayudante de investigación, se informaba de los objetivos del estudio (estudio sobre dinámica de la vida marital o de convivencia por medio de un cuestionario), del responsable del mismo (autor del artículo) y criterios de inclusión (ser mayor de edad, estar casado o en unión libre con una persona del sexo opuesto y saber leer y escribir). Si la persona deseaba participar voluntariamente y satisfacía los criterios de inclusión, se dejaba un cuestionario para recogerlo media hora después o cuando lo indicase la persona. Se instruía que debía contestarlo a solas, sin que su pareja leyese las preguntas o respuestas. Al recogerlo se preguntaba si la pareja había estado presente a la hora de 
contestar y había visto sus contestaciones. En caso de que sí, se descartaba el caso. En caso de que no, se revisaba que estuviera todo el cuestionario contestado. Si había preguntas vacías, se pedía amablemente que se respondiesen y se explicaba que era necesario para que el análisis de los datos fuese confiable.

Los entrevistadores fueron alumnos de primer semestre de la facultad de psicología de la universidad Autónoma de Nuevo León que cursaban la materia de Investigación en Psicología. Recibieron entrenamiento por parte del responsable del proyecto de investigación para colectar los casos y fueron compensados con puntos extras en su calificación. La muestra fue reclutada de enero a mayo de 2014.

\section{Análisis de datos}

En relación con el primer objetivo de estudiar las propiedades de los ítems, se describieron sus distribuciones, usando la mediana $(M d n)$ como estadístico de tendencia central, el rango semi-intercuartílico $\left(R S I=\left[Q_{3}-Q_{1}\right] / 2\right)$ como estadístico de variación, y el coeficiente intercuartílico de asimetría $\left(A_{I}=\left[Q_{3}+Q_{1-} 2^{*} Q_{2}\right] /\left[Q_{3}-Q_{1}\right]\right)$ y el coeficiente de curtosis percentílico corregido $\left(C_{p c}=\left(R S I /\left[P_{90}-P_{10}\right]\right)-0.263\right)$ como estadísticos de la forma de la distribución. La discriminación se determinó por la diferencia significativa de tendencia central en el ítem entre los grupos de puntuaciones altas y bajas en la escala. Las puntuaciones en la escala se crearon por la suma simple de los ítems (invirtiendo las puntuaciones de los dos ítems redactados en sentido opuesto al rasgo). Los grupos se definieron por el primer cuartil y el tercero en la escala. La diferencia de tendencia central se contrastó por la prueba $U$ de Mann-Whitney. La consistencia interna se estimó por la correlación del ítem con el resto de la escala, por el efecto de la eliminación del ítem sobre la consistencia interna de la escala y la comunalidad tras la extracción de un factor por el 
método de Mínimos Cuadrados No Ponderados (ULS). La correlación se calculó por el coeficiente de correlación poliserial ( $r_{P S}[$ ítem, test-ítem]) y la consistencia interna por el coeficiente alfa ordinal $\left(\alpha\right.$ ordinal [test-ítem]). Si $r_{P S}[$ ítem, test-ítem] $\geq .5$, disminuía la consistencia interna de la escala con la eliminación del ítem ( $\alpha$ ordinal [test-ítem] $<\alpha$ ordinal [test]) y la comunalidad del ítem $\geq .25$, en ese caso se consideró que el ítem presentaba consistencia interna buena.

En relación con el segundo objetivo de calcular la consistencia interna de la escala se usó el coeficiente alfa ordinal ( $\alpha$ ordinal) y la consistencia compuesta por el coeficiente $\rho$ de Joreskog. A semejanza del coeficiente alfa de Cronbach se interpretó que valores de $\alpha$ ordinal y $\rho$ de Joreskog $<.5$ evidencian una consistencia interna inaceptable, de .5 a .59 pobre, de .6 a .69 cuestionable, de .7 a .79 aceptable, de .8 a .89 buena y $\geq .9$ excelente (Elosua \& Zumbo, 2008; Yang \& Green, 2010). Además se calculó el promedio de la varianza extraída (AVE). Un valor mayor que .5 es indicador de consistencia interna y validez de constructo unidimensional (Fornell \& Larker, 1981).

En relación con el tercer objetivo de determinar el número de factores, desde la matriz de correlaciones policóricas, se realizó el análisis paralelo de Horn (percentil 95 como criterio de intersección, datos generados por permutaciones con 500 muestras simuladas), se hizo el cálculo de promedios de las correlaciones parciales al cuadrado (promedio mínimo de Velicer) y el análisis de coordenadas óptimas (incremento en el gradiente del autovalor con respecto al gradiente del anterior autovalor) (Courtney, 2013).

Las correlaciones policóricas $\left(r_{P C}\right)$ se calcularon por el método de Dos Pasos para Máxima Verosimilitud. Los valores de $r_{P C}<.3$ se consideraron correlaciones bajas, de .3 a .49 moderadas, de .5 a .69 altas, de .7 a .89 muy altas y $\geq .9$ unitarias (Hoffmann et al., 
2013). La estabilidad de las estimaciones se determinó por un error estándar <.1, límites con un intervalo de confianza del $95 \%$ dentro del rango admisible $[-1,1]$ y una matriz de correlaciones definida positiva. El supuesto de normalidad bivariada se contrastó por la prueba chi-cuadrado de la razón de verosimilitud $\left(\chi^{2}\right)$ y dos índices: cociente entre $\chi^{2}$ y sus grados de libertad $\left(\chi^{2} / g l\right)$ y el error de aproximación cuadrático medio (RMSEA). Un muy buen ajuste vendría indicado por un valor $p>.05$ en la prueba $\chi^{2}, \chi^{2} / g l \leq 2$ y $R M S E A \leq .05$, bueno por un valor $p>.01$ en la prueba $\chi^{2}, \chi^{2} / g l \leq 2$ y $R M S E A \leq .05$, un ajuste aceptable por un valor $p<.01$ en la prueba $\chi^{2}, \chi^{2} / g l \leq 3$ y $R M S E A \leq .05$ y malo por un valor $p<.01$ en la prueba $\chi^{2}, 5>\chi^{2} / \mathrm{gl}>3$ y $.08>$ RMSEA $>.05$ y muy malo un valor $p<.01$ en la prueba $\chi^{2}, \chi^{2} / \mathrm{gl}>5$ y $R M S E A>.08$ (Joreskog \& Sörbom, 2000).

En relación con el cuarto objetivo de validar el modelo unidimensional, se aplicó análisis factorial confirmatorio sobre la muestra conjunta (análisis unigrupo). Para contrastar su invarianza entre ambos sexos, se realizó un análisis multigrupo (sobre la muestra de mujeres y sobre la de hombres). En este último análisis, se definieron cuatro modelos anidados en restricciones (especificación de equivalencia de parámetros entre las dos muestras): sin restricciones (parámetros libres en las dos muestras), con restricciones en los pesos de medida (equivalencia en $\lambda_{\mathrm{i}}$ entre las dos muestras), con restricciones adicionales en la varianza estructural $\left(\Theta_{\xi}\right)$ y con restricciones adicionales en los residuos de medida $\left(\delta_{\mathrm{i}}\right)$.

Desde la matriz de correlaciones policóricas, la función de discrepancia se estimó por Mínimos Cuadrados No Ponderados (ULS) en ambos análisis. El ajuste se valoró usando la chi-cuadrada relativa $\left(\chi^{2} / g l\right)$, el índice de bondad de ajuste sin ajustar $(G F I)$ y ajustado (AGFI), el índice normado de ajuste $(N F I)$, el índice relativo de ajuste o 
coeficiente $\rho_{1}$ de Bollen (RFI) y el residuo estandarizado cuadrático medio (SRMR). Siguiendo a Byrne (2010), $\chi^{2} / g l \leq 2, G F I, R F I$ y $N F I \geq .95, A G F I \geq .9$ y $S R M R \leq .05$ reflejan un ajuste bueno; y $\chi^{2} / g l \leq 3, G F I, R F I$ y $N F I \geq .9, A G F I \geq .85$ y $S R M R \leq .1$ reflejan un ajuste aceptable.

Se estimó la parsimonia por la razón de parsimonia de James-Mulaik-Brett $(R P)$. Unos valores de $R P$ entre 0 a .24 se interpretan como parsimonia baja, de .25 a .49 mediabaja, de .50 a .74 media-alta $y \geq .75$ alta (James, Mulaik, \& Brett, 1982). La significación de parámetros se estimó por el método de percentiles corregidos de sesgo, extrayendo 2,000 muestras. Al ser los datos de entrada correlaciones, se requirió que la matriz fuese definida positiva y asumir una distribución teórica; consecuente con el hecho de ser correlaciones policóricas, se asumió distribución normal (remuestreo paramétrico) (Bowen \& Guo, 2011).

En relación con el quinto objetivo, la exploración y el contraste modelos factoriales alternos al de un factor no fueron necesarios por la convergencia de los criterios empíricos para determinar el número de factores en un factor y los resultados del análisis factorial confirmatorio unigrupo y multigrupo (ambos sexos) con el modelo de un factor. Los cálculos se realizaron con SPSS22, menú R versión 2.4, LISREL versión 8.52 y con AMOS versión 16.

\section{Resultados}

\section{Propiedades de los ítems y consistencia interna de la RAS}

No se presentó efecto suelo (el valor mínimo aparece en el 90\% o más de los casos) ni efecto techo (el valor máximo aparece en el $90 \%$ o más de los casos) en la distribución 
de ninguno de los siete ítems. Tampoco ningún ítem mostro variación reducida $(R S I<0.5)$, como consecuencia de la combinación de asimetría y apuntamiento (véase Tabla 2).

Tabla 2

Distribución de los siete ítems de la RAS

\begin{tabular}{|c|c|c|c|c|c|c|c|c|c|c|c|}
\hline \multirow{3}{*}{ Ítems } & \multicolumn{5}{|c|}{ Escala de respuesta } & \multicolumn{6}{|c|}{ Descriptivos } \\
\hline & 1 & 2 & 3 & 4 & 5 & & artil & & & & \\
\hline & $f(\%)$ & $f(\%)$ & $f(\%)$ & $f(\%)$ & $f(\%)$ & $Q_{1}$ & $Q_{2}$ & $Q_{3}$ & & & \\
\hline RAS1 & $\begin{array}{c}24 \\
(3.0 \%)\end{array}$ & $\begin{array}{c}35 \\
(4.3 \%)\end{array}$ & $\begin{array}{c}169 \\
(20.9 \%)\end{array}$ & $\begin{array}{c}376 \\
(46.6 \%)\end{array}$ & $\begin{array}{c}203 \\
(25.2 \%)\end{array}$ & 3 & 4 & 5 & 1 & 0 & .237 \\
\hline RAS2 & $\begin{array}{c}10 \\
(1.2 \%)\end{array}$ & $\begin{array}{c}45 \\
(5.6 \%)\end{array}$ & $\begin{array}{c}131 \\
(16.2 \%)\end{array}$ & $\begin{array}{c}344 \\
(42.6 \%)\end{array}$ & $\begin{array}{c}277 \\
(34.3 \%)\end{array}$ & 4 & 4 & 5 & 0.5 & 1 & -.013 \\
\hline RAS3 & $\begin{array}{c}9 \\
(1.1 \%)\end{array}$ & $\begin{array}{c}48 \\
(5.9 \%)\end{array}$ & $\begin{array}{c}143 \\
(17.7 \%)\end{array}$ & $\begin{array}{c}316 \\
(39.2 \%)\end{array}$ & $\begin{array}{c}291 \\
(36.1 \%)\end{array}$ & 4 & 4 & 5 & 0.5 & 1 & -.013 \\
\hline RAS4 & $\begin{array}{c}418 \\
(51.8 \%)\end{array}$ & $\begin{array}{c}292 \\
(36.2 \%)\end{array}$ & $\begin{array}{c}52 \\
(6.4 \%)\end{array}$ & $\begin{array}{c}27 \\
(3.3 \%)\end{array}$ & $\begin{array}{c}18 \\
(2.2 \%)\end{array}$ & 1 & 1 & 2 & 0.5 & 1 & -.013 \\
\hline RAS5 & $\begin{array}{c}17 \\
(2.1 \%)\end{array}$ & $\begin{array}{c}59 \\
(7.3 \%)\end{array}$ & $\begin{array}{c}184 \\
(22.8 \%)\end{array}$ & $\begin{array}{c}336 \\
(41.6 \%)\end{array}$ & $\begin{array}{c}211 \\
(26.1 \%)\end{array}$ & 3 & 4 & 5 & 1 & 0 & .237 \\
\hline RAS6 & $\begin{array}{c}10 \\
(1.2 \%)\end{array}$ & $\begin{array}{c}30 \\
(3.7 \%)\end{array}$ & $\begin{array}{c}83 \\
(10.3 \%)\end{array}$ & $\begin{array}{c}281 \\
(34.8 \%)\end{array}$ & $\begin{array}{c}403 \\
(49.9 \%)\end{array}$ & 4 & 4 & 5 & 0.5 & 1 & -.013 \\
\hline RAS7 & $\begin{array}{c}170 \\
(21.1 \%)\end{array}$ & $\begin{array}{c}129 \\
(16.0 \%)\end{array}$ & $\begin{array}{c}413 \\
(51.2 \%)\end{array}$ & $\begin{array}{c}80 \\
(9.9 \%)\end{array}$ & $\begin{array}{c}15 \\
(1.9 \%)\end{array}$ & 2 & 3 & 3 & 0.5 & -1 & -.096 \\
\hline
\end{tabular}

Nota. $R S I=$ rango semi-intercuartílico, $A_{I}=$ asimetría intercuartílica. $C_{p c}=$ coeficiente de curtosis

Percentílico corregido. 
La mediana en los cinco ítems directos coincidió en cuatro, que corresponde a una respuesta de bastante satisfacción. Tres de los cinco ítems directos (ítem 2 "en general, ¿hasta qué punto está satisfechalo con su relación?”, ítem 3 “qqué tan buena es su relación en comparación con la mayoría?” e ítem 6 “¿cuánto ama a su pareja?”) mostraron asimetría positiva $(C A I=1)$, al coincidir la mediana $\left(Q_{2}=4\right)$ con el primer cuartil $\left(Q_{l}=4\right)$; mesocurtosis $(C p c=-.01)$; y variación intermedia $(R S I=0.5)$. La mayoría de las personas encuestadas indicaron que están bastante o totalmente satisfechos en estos tres ítems (véase Tabla 2).

Las distribuciones de los otros dos ítems directos (ítems 1 "¿de qué manera considera usted que su pareja satisface sus necesidades?” e ítem 5 “ ¿hasta qué punto su relación satisface sus expectativas iniciales?") mostraron simetría $(C A I=0)$, al quedar la mediana $\left(Q_{2}=4\right)$ entre el primer cuartil $\left(Q_{1}=3\right)$ y el tercero $\left(Q_{3}=5\right)$; apuntamiento $(C p c=$ .24), al contar con colas alargadas hacia ambos extremos (1 y 5) y mayor concentración de valores en torno a la mediana que un perfil mesocúrtico; y variación amplia como consecuencia del apuntamiento $(R S I=1)$. Aunque la mayoría de los encuestados indicaron que están bastante o totalmente satisfechos, el rango de respuesta resultó más amplio y hubo más casos extremos que en los otros tres ítems directos (véase Tabla 2).

La mediana del ítem inverso 4 (“¿con qué frecuencia desea no haberse implicado con su pareja?") fue dos, que corresponde a una respuesta de bastante desacuerdo con el arrepentimiento. Su distribución mostró asimetría positiva $(C A I=1)$; mesocurtosis $(C p c=$ $.01)$; y variación intermedia $(R S I=0.5)$. La mayoría de los participantes indicaron estar bastante o totalmente satisfechos con su decisión de casarse (véase Tabla 2). 
La mediana del ítem inverso 7 (“¿cuántos problemas hay en su relación de pareja?") fue tres, que corresponde a una respuesta término medio. Su distribución mostró asimetría negativa $(C A I=-1)$, al coincidir la mediana $\left(Q_{2}=3\right)$ con el tercer cuartil $\left(Q_{3}=3\right)$; ligero aplanamiento $(C p c=-.1)$, al contar con colas cortas hacia ambos extremos y menor concentración de valores en torno a la mediana que un perfil mesocúrtico; y variación intermedia $(R S I=0.5)$. Pocos participantes indicaron conflictos frecuentes (véase Tabla 2).

Los siete ítems fueron discriminativos $(p<.001)$ y tuvieron correlaciones con el resto de la escala mayores que .5 , variando de .53 a .84 con una media de .75 en valores absolutos. La consistencia interna de los siete ítems fue alta $(\alpha$ ordinal $=.93)$ y se incrementó una décima con la eliminación del ítem inverso 7 “¿cuántos problemas hay en su relación de pareja?" ( $\alpha$ ordinal $[t-i]=.94)$. Al extraer un factor, las cargas factoriales en valores absolutos variaron de .56 a .92 con una media de .81 , así las comunalidades variaron de .31 a .84 con un promedio de .67 (véase Tabla 3 ). 
Tabla 3

Discriminación y consistencia interna de los siete ítems de la RAS

\begin{tabular}{ccccccc}
\hline \multirow{2}{*}{ Ítems } & Discriminación & \multicolumn{2}{c}{ Consistencia interna } & \multicolumn{3}{c}{ AFE } \\
\cline { 2 - 7 } & $Z_{U}$ & $r_{P S}(i, t-i)$ & $\alpha$ ordinal $(t-i)$ & $\lambda$ & $\lambda^{2}$ & $U^{2}$ \\
\hline RAS1 & $-17.405^{* * *}$ & $.760^{* * * *}$ & .919 & .846 & .716 & .284 \\
RAS2 & $-18.590^{* * * *}$ & $.841^{* * *}$ & .911 & .915 & .838 & .162 \\
RAS3 & $-18.529^{* * * *}$ & $.810^{* * * *}$ & .914 & .890 & .792 & .208 \\
RAS4 & $-17.958^{* * * *}$ & $-.671^{* * *}$ & .926 & -.720 & .518 & .482 \\
RAS5 & $-18.356^{* * * *}$ & $.822^{* * *}$ & .913 & .891 & .794 & .206 \\
RAS6 & $-18.077^{* * *}$ & $.780^{* * *}$ & .916 & .856 & .733 & .267 \\
RAS7 & $-14.689^{* * *}$ & $-.532^{* * *}$ & .941 & -.555 & .308 & .692 \\
\hline
\end{tabular}

Nota. $* * * p<.001 . Z_{U}=$ Valor estandarizado del estadístico U de Mann-Whitney. $r_{P S}(i, t-i)=$ correlación poliserial del ítem con el resto de la escala. $\alpha \operatorname{ordinal}(t-i)=$ coeficiente alfa ordinal de la escala sin incluir al ítem. AFE = Extracción de un factor por Mínimos Cuadrados No Ponderados: $\lambda=$ carga factorial, $\lambda^{2}=$ comunalidad, $U^{2}=$ unicidad.

Al igual que en la muestra conjunta, la consistencia interna por el coeficiente alfa ordinal fue excelente tanto en la muestra de mujeres $(\alpha$ ordinal $=.94)$ como en la muestra de hombres $(\alpha$ ordinal $=.92)$. Si se hubiera estimado por el coeficiente alfa de Cronbach, la consistencia interna hubiera sido buena en la muestra conjunta $(\alpha$ de Cronbach $=.89)$ y en la de hombres $(\alpha$ de Cronbach $=.88)$ y excelente en la de mujeres $(\alpha$ de Cronbach $=.91)$. 


\section{Número de factores desde la matriz de correlaciones policóricas}

Las correlaciones policóricas (en valores absolutos) entre los siete ítems variaron de .43 a .83 con una media .66. En todos los casos, al ser estimadas con un intervalo de confianza del 95\%, sus límites quedaron en un rango admisible [-1, 1]. La matriz de correlaciones policóricas fue definida positiva. Los errores estándar de las 21 correlaciones policóricas variaron de .01 a .03 con una media de .02, lo que indica que las estimaciones fueron estables y admisibles. En las 21 correlaciones, la bondad de ajuste del modelo de distribución bivariada normal se sostuvo por el índice de error de aproximación cuadrático medio $(R M S E A \leq .05, p>.1)$, aun reduciendo el intervalo de confianza al $90 \%$ para compensar la tendencia al error tipo 2 (mantener la hipótesis nula siendo falsa) de la prueba, especialmente con un tamaño de muestra $\geq 400$. El cociente entre $\chi^{2}$ y sus grados de libertad fue $\leq 2$ en 6 de las 21 correlaciones (28.6\%), fue $<3$ en $13(61.9 \%)$ y fue $<5$ en las 21 correlaciones. Por la prueba $\chi^{2}$, el supuesto de normalidad bivariada se sostendría en dos correlaciones con un valor $p>.05$ y en siete con un valor $p>.01$, al ampliar el intervalo de confianza al 99\% para compensar la tendencia al error tipo 1 (rechazar la hipótesis nula siendo verdadera) de la prueba, especialmente con un tamaño de muestra $\geq 400$. En 2 de las 21 correlaciones (10\%) habría muy buen ajuste, en 4 (19\%) buen ajuste, en 7 (33\%) aceptable, en 8 (38\%) malo y en ninguna de las 21 correlaciones muy malo (véase Tabla 4). 
Tabla 4

Correlaciones policóricas y contraste de la normalidad bivariada en la muestra conjunta

\begin{tabular}{|c|c|c|c|c|c|c|c|}
\hline \multicolumn{3}{|c|}{ Correlación policórica } & \multicolumn{5}{|c|}{ Bondad de ajuste } \\
\hline Ítems & $r_{P C}[\mathrm{IC} 95 \%]$ & $E E$ & $\chi^{2}$ & $p^{*}$ & $\chi^{2} / g l$ & RMSEA & $p^{* *}$ \\
\hline RAS2 y RAS1 & $.831[.806, .856]$ & .013 & 43.457 & $<.001$ & 2.897 & .048 & 1 \\
\hline RAS3 y RAS1 & $.750[.715, .785$ & .018 & 23.573 & .073 & 1.572 & .027 & 1 \\
\hline RAS3 y RAS2 & $.812[.783,0.841]$ & .015 & 26.099 & .037 & 1.740 & .030 & 1 \\
\hline RAS4 y RAS1 & $-.541[-.600,-.482]$ & .030 & 59.771 & $<.001$ & 3.985 & .061 & 1 \\
\hline RAS4 y RAS2 & $-.642[-.691,-.593]$ & .025 & 72.567 & $<.001$ & 4.838 & .069 & 999 \\
\hline RAS4 y RAS3 & $-.623[-.674,-.572]$ & .026 & 43.919 & $<.001$ & 2.928 & .049 & 1 \\
\hline RAS5 y RAS1 & $.736[.701, .771]$ & .018 & 50.373 & $<.001$ & 3.358 & .054 & 1 \\
\hline RAS5 y RAS2 & $.794[.765, .823]$ & .015 & 28.748 & .017 & 1.917 & .034 & 1 \\
\hline RAS5 y RAS3 & $.817[.790, .844]$ & .014 & 26.698 & .031 & 1.780 & .031 & 1 \\
\hline RAS5 y RAS4 & $-.650[-.697,-.603]$ & .024 & 67.392 & $<.001$ & 4.493 & .066 & 1 \\
\hline RAS6 y RAS1 & $.699[.656, .742]$ & .022 & 23.270 & .079 & 1.551 & .026 & 1 \\
\hline RAS6 y RAS2 & $.777[.742, .812]$ & .018 & 30.603 & .010 & 2.040 & .036 & 1 \\
\hline RAS6 y RAS3 & $.752[.715, .789]$ & .019 & 26.233 & .036 & 1.749 & .030 & 1 \\
\hline RAS6 y RAS4 & $-.672[-.719,-.625]$ & .024 & 45.206 & $<.001$ & 3.014 & .050 & 1 \\
\hline RAS6 y RAS5 & $.782[.749, .815]$ & .017 & 30.187 & .011 & 2.012 & .035 & 1 \\
\hline RAS7 y RAS1 & $-.429[-.494,-.364]$ & .033 & 40.321 & $<.001$ & 2.688 & .046 & 1 \\
\hline RAS7 y RAS2 & $-.496[-.557,-.435]$ & .031 & 47.645 & $<.001$ & 3.176 & .052 & 1 \\
\hline RAS7 y RAS3 & $-.473[-.536,-.410]$ & .032 & 38.774 & .001 & 2.585 & .044 & 1 \\
\hline
\end{tabular}




\begin{tabular}{ccccccccc}
\hline \multicolumn{3}{c}{ Correlación policórica } & \multicolumn{6}{c}{ Bondad de ajuste } \\
\hline Ítems & $r_{P C}[$ IC 95\%] & $E E$ & $\chi^{2}$ & $p^{*}$ & $\chi^{2} / g l$ & $R M S E A$ & $p^{* *}$ \\
& & & & & & & \\
\hline RAS7 y RAS4 & $.570[.513, .627]$ & .029 & 59.881 & $<.001$ & 3.992 & .061 & 1 \\
RAS7 y RAS5 & $-.498[-.557,-.439]$ & .030 & 70.911 & $<.001$ & 4.727 & .068 & 1 \\
RAS7 y RAS6 & $-.469[-.534,-.404]$ & .033 & 40.818 & $<.001$ & 2.721 & .046 & 1 \\
\hline
\end{tabular}

*Hipótesis nula: ajuste a una distribución bivariada normal. Grados de libertad $(g l)=15$.

** Hipótesis nula: RMSEA $\leq .05$.

El número de factores convergió en uno por el criterio de Horn, de Velicer y de coordenadas óptimas, incluso también por el de Kaiser (véase Figura 2).

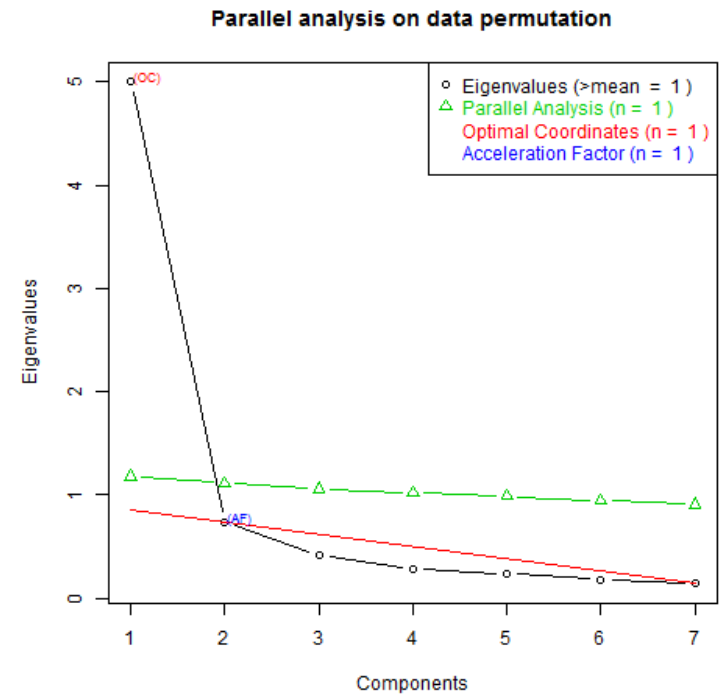

Figura 2. Curvas de sedimentación para el análisis paralelo de Horn.

\section{Contraste de modelo unidimensional en la muestra conjunta (análisis unigrupo)}

Se contrastó un modelo unidimensional con siete indicadores y sus siete residuos de medida independientes entre sí. La solución factorial fue admisible. Los pesos de medida variaron de .57 a .91 con un promedio .81 (véase Figura 3). El ajuste fue bueno por los seis 
índices contemplados. La parsimonia del modelo fue media-alta $(R P=.67)$. $\mathrm{Al}$ asumir normalidad multivariada en concordancia con las correlaciones policóricas, todos los parámetros serían significativos por la prueba de percentiles corregidos de sesgo (remuestreo paramétrico), y la bondad de ajuste se mantendría con una probabilidad mayor que .01 en la prueba chi-cuadrado de la razón de verosimilitud $\left(\chi^{2}[14, N=807]=26.35, p\right.$ $=.020)$ (véase Tabla 5). Desde los pesos de medida, la consistencia compuesta por el coeficiente rho de Joreskog fue buena $(\rho=.80)$ y el promedio de la varianza extraída fue mayor que $.5(\mathrm{AVE}=.67)$.

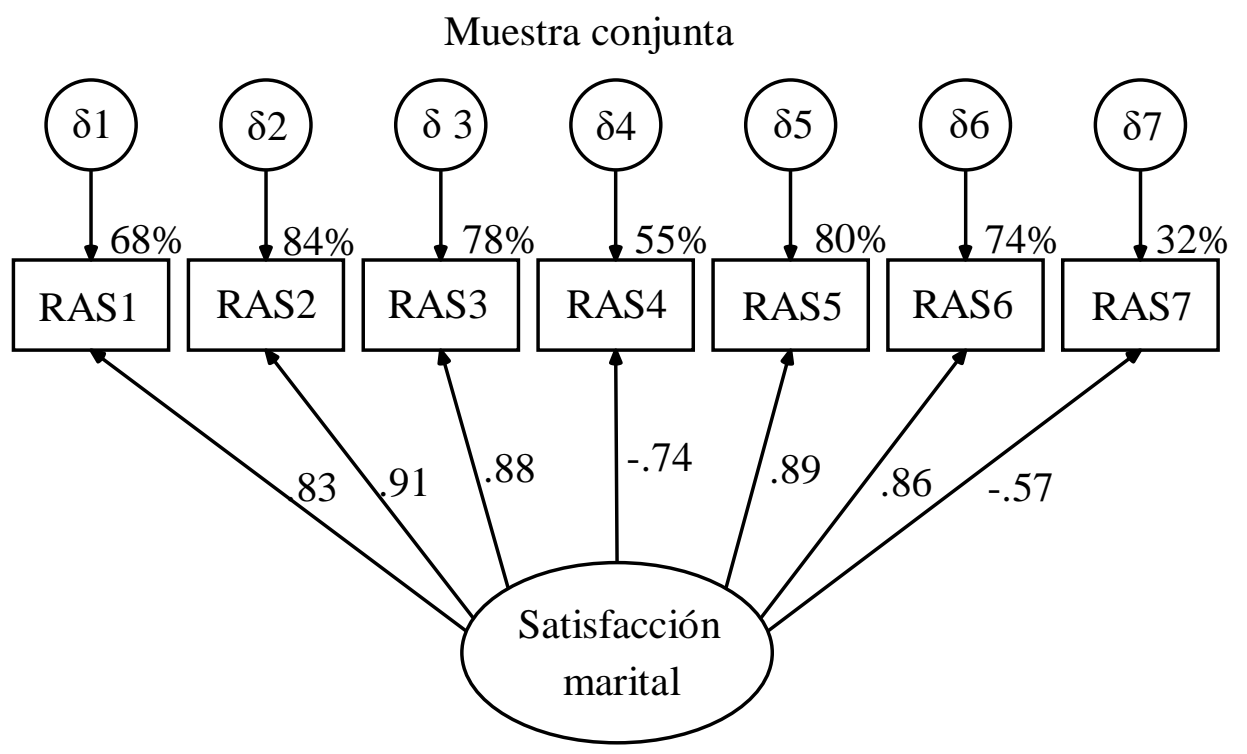

Figura 3. Modelo de un factor con siete indicadores con los coeficientes estandarizados estimados por Mínimos Cuadrados No Ponderados en la muestra conjunta. 


\section{Tabla 5}

Índices de ajuste del modelo de un factor en el análisis unigrupo y multigrupo

\begin{tabular}{|c|c|c|c|c|c|c|c|c|c|c|}
\hline \multirow{3}{*}{ IA } & \multicolumn{5}{|c|}{ Modelo de un factor } & \multicolumn{5}{|c|}{ Modelo de un factor modificado } \\
\hline & \multirow{2}{*}{$\begin{array}{l}\text { Uni- } \\
\text { grupo }\end{array}$} & \multicolumn{4}{|c|}{ Multigrupo (sexos) } & \multirow{2}{*}{$\begin{array}{l}\text { Uni- } \\
\text { grupo }\end{array}$} & \multicolumn{4}{|c|}{ Multigrupo (sexos) } \\
\hline & & SR & $\mathrm{PM}$ & $\mathrm{VE}$ & $\mathrm{RM}$ & & SR & $\mathrm{PM}$ & $\mathrm{VE}$ & $\mathrm{RM}$ \\
\hline$\chi^{2}$ & 18.929 & 29.913 & 36.270 & 107.301 & 114.710 & 8.014 & 10.056 & 17.994 & 88.123 & 96.011 \\
\hline$g l$ & 14 & 28 & 34 & 35 & 42 & 13 & 26 & 32 & 33 & 41 \\
\hline$\chi^{2} / g l$ & 1.924 & 1.068 & 1.067 & 3.066 & 2.731 & 0.616 & 0.387 & 0.562 & 2.670 & 2.342 \\
\hline$p$ & .020 & .367 & .363 & $<.001$ & $<.001$ & .843 & .998 & .978 & $<.001$ & $<.001$ \\
\hline GFI & .997 & .996 & .995 & .987 & .986 & .999 & .999 & .998 & .989 & .988 \\
\hline$A G F I$ & .993 & .992 & .992 & .978 & .981 & .998 & .997 & .996 & .981 & .984 \\
\hline$N F I$ & .995 & .995 & .994 & .981 & .980 & .999 & .998 & .997 & .985 & .983 \\
\hline$R F I$ & .993 & .992 & .992 & .978 & .980 & .998 & .997 & .996 & .981 & .983 \\
\hline$S R M R$ & .039 & .053 & .058 & .093 & .073 & .022 & .018 & .024 & .063 & .046 \\
\hline$R P$ & .667 & .667 & .810 & .833 & 1 & .619 & .619 & .762 & .786 & .976 \\
\hline$\Delta \chi^{2}$ & & & & & & 10.915 & 19.857 & 18.276 & 19.178 & 18.699 \\
\hline$\Delta g l$ & & & & & & 1 & 2 & 2 & 2 & 1 \\
\hline$p$ & & & & & & .001 & $<.001$ & $<.001$ & $<.001$ & $<.001$ \\
\hline
\end{tabular}

Nota. Método: Mínimos Cuadrados No Ponderados. Modelos anidados en restricciones: SR $=\sin$ restricciones, $\mathrm{PM}=$ en los pesos de medida, $\mathrm{VE}=$ en la varianza estructural y $\mathrm{RM}=$ en los residuos de medida. $\Delta \chi^{2}$ y $\Delta g l=$ comparación con el correspondiente modelo sin modificar.

Si se introdujera una correlación entre los residuos de medida del ítem inverso 4 “con qué frecuencia desea no haberse implicado con su pareja?” y del ítem inverso 7 “¿cuántos problemas hay en su relación de pareja?” ( $\left.\psi_{\delta 4 \delta 7}\right)$, el ajuste mejoraría y se conservaría una parsimonia media-alta $(R P=.62)$ (véase Tabla 5). Al asumir normalidad 
multivariada en concordancia con las correlaciones policóricas, todos los parámetros serían significativos por la prueba de percentiles corregidos de sesgo (remuestreo paramétrico), incluyendo la correlación entre los dos residuos $\left(\psi_{\delta 4 \delta 7}=.32\right.$, IC 95\%: $\left..25, .38, p=.001\right)$; el incremento en la bondad de ajuste sería estadísticamente significativa $\left(\Delta \chi^{2}[1, N=807]=\right.$ $10.92, p=.001)$; y la bondad de ajuste se mantendría con una probabilidad mayor que .05 en la prueba chi-cuadrado de la razón de verosimilitud $\left(\chi^{2}[13, N=807]=8.01, p=.843\right)$.

\section{Invarianza del modelo unidimensional entre ambos sexos (análisis multigrupo)}

Se contrastó la invarianza del modelo de un factor con siete indicadores (con los siete residuos independientes) entre ambos sexos (análisis multigrupo).

En la muestra de hombres, las correlaciones policóricas (en valores absolutos) variaron de .33 a .81 con una media .61. En todos los casos, al ser estimadas con un intervalo de confianza del 95\%, sus límites quedaron en un rango admisible $[-1,1]$. La matriz de correlaciones policóricas fue definida positiva. Los errores estándar de las 21 correlaciones policóricas variaron de .02 a .05 con una media de .04 , lo que indica que las estimaciones fueron estables y admisibles. En las 21 correlaciones, la bondad de ajuste del modelo de distribución bivariada normal se sostuvo por el índice de error de aproximación cuadrático medio $(R M S E A \leq .05, p>.1)$. El cociente entre $\chi^{2}$ y sus grados de libertad fue $\leq$ 2 en 7 de las 21 correlaciones $(33.3 \%)$, fue $<3$ en $20(95.2 \%)$ y fue $<5$ en las 21 correlaciones. Por la prueba $\chi^{2}$, el modelo de distribución bivariada normal subyacente se sostuvo en seis correlaciones con un valor $p>.05$; y en ocho con un valor $p>.01$. En 6 de las 21 correlaciones $(28.6 \%)$ habría muy buen ajuste, en $1(4.8 \%)$ buen ajuste, en 13 (61.8\%) aceptable, en 1 (4.8\%) malo y en ninguna muy malo (véase Tabla 6). 
Tabla 6

Correlaciones policóricas y contraste de la normalidad bivariada en hombres

\begin{tabular}{|c|c|c|c|c|c|c|c|}
\hline \multicolumn{3}{|c|}{ Correlaciones policóricas } & \multicolumn{5}{|c|}{ Bondad de ajuste } \\
\hline Ítems & $r_{P C}[\mathrm{IC} 95 \%]$ & $E E$ & $\chi^{2}$ & $p^{*}$ & $\chi^{2} / g l$ & RMSEA & $p^{* *}$ \\
\hline RAS2 y RAS1 & $.800[.757, .843]$ & .022 & 32.080 & .006 & 2.139 & .055 & .998 \\
\hline RAS3 y RAS1 & $.674[.609, .739]$ & .033 & 31.992 & .006 & 2.133 & .055 & .998 \\
\hline RAS3 y RAS2 & $.805[.758, .852]$ & .024 & 20.507 & .153 & 1.367 & .031 & 1 \\
\hline RAS4 y RAS1 & $-.438[-.536,-.340]$ & .050 & 40.299 & $<.001$ & 2.687 & .067 & .985 \\
\hline RAS4 y RAS2 & $-.611[-.687,-.535]$ & .039 & 39.296 & .001 & 2.620 & .066 & .988 \\
\hline RAS4 y RAS3 & $-.521[-.609,-.433]$ & .045 & 31.460 & .008 & 2.097 & .054 & .999 \\
\hline RAS5 y RAS1 & $.648[.581, .715]$ & .034 & 43.733 & $<.001$ & 2.916 & .071 & .971 \\
\hline RAS5 y RAS2 & $.776[.727, .825]$ & .025 & 15.421 & .422 & 1.028 & .009 & 1 \\
\hline RAS5 y RAS3 & $.766[.715, .817]$ & .026 & 24.448 & .058 & 1.630 & .041 & 1 \\
\hline RAS5 y RAS4 & $-.592[-.668,-.516]$ & .039 & 41.063 & $<.001$ & 2.738 & .068 & .983 \\
\hline RAS6 y RAS1 & $.644[.573, .715]$ & .036 & 30.275 & .011 & 2.018 & .052 & .999 \\
\hline RAS6 y RAS2 & $.747[.692, .802]$ & .028 & 31.380 & .008 & 2.092 & .054 & .999 \\
\hline RAS6 y RAS3 & $.691[.626, .756]$ & .033 & 28.428 & .019 & 1.895 & .049 & .999 \\
\hline RAS6 y RAS4 & $-.628[-.702,-.554]$ & .038 & 33.730 & .004 & 2.249 & .058 & .997 \\
\hline RAS6 y RAS5 & $.758[.705, .811]$ & .027 & 32.774 & .005 & 2.185 & .056 & .998 \\
\hline RAS7 y RAS1 & $-.325[-.429,-.221]$ & .053 & 35.831 & .002 & 2.389 & .061 & .995 \\
\hline RAS7 y RAS2 & $-.465[-.557,-.373]$ & .047 & 40.159 & $<.001$ & 2.677 & .067 & .986 \\
\hline RAS7 y RAS3 & $-.420[-.516,-.324]$ & .049 & 12.866 & .613 & 0.858 & $<.001$ & 1 \\
\hline
\end{tabular}




\begin{tabular}{cccccccc}
\hline \multicolumn{3}{c}{ Correlaciones policóricas } & \multicolumn{5}{c}{ Bondad de ajuste } \\
\hline Ítems & $r_{P C}[$ IC 95\%] & $E E$ & $\chi^{2}$ & $p^{*}$ & $\chi^{2} / g l$ & $R M S E A$ & $p^{* *}$ \\
& & & & & & & \\
\hline RAS7 y RAS4 & $.566[.482, .650]$ & .043 & 15.958 & .385 & 1.064 & .013 & 1 \\
& & & & & & & \\
RAS7 y RAS5 & $-.451[-.541,-.361]$ & .046 & 47.675 & $<.001$ & 3.178 & .076 & .943 \\
RAS7 y RAS6 & $-.405[-.507,-.303]$ & .052 & 22.838 & .088 & 1.523 & .037 & 1 \\
\hline
\end{tabular}

*Ajuste a una distribución bivariada normal. Grados de libertad $(g l)=15$. ** RMSEA $\leq .05$.

En la muestra de mujeres, las correlaciones policóricas (en valor absoluto) variaron de .52 a .86 con una media .70 . En todos los casos, al ser estimadas con un intervalo de confianza del 95\%, sus límites quedaron en un rango admisible $[-1,1]$. La matriz de correlaciones policóricas fue definida positiva. Los errores estándar de las 21 correlaciones policóricas variaron de .02 a .04 con una media de .03 , lo que indica que las estimaciones fueron estables y admisibles. En las 21 correlaciones, la bondad de ajuste del modelo de distribución bivariada normal se sostuvo por el índice de error de aproximación cuadrático medio $(R M S E A \leq .05, p>.1)$. El cociente entre $\chi^{2}$ y sus grados de libertad fue $\leq 2$ en 13 de las 21 correlaciones (61.9\%), fue $<3$ en $19(90.5 \%)$ y fue $<5$ en las 21 correlaciones. Por la prueba $\chi^{2}$, el modelo de distribución bivariada normal subyacente se sostuvo en 11 correlaciones con un valor $p>.05$; y en 13 con un valor $p>.01$. En 11 de las 21 correlaciones (52.4\%) habría muy buen ajuste, en 2 (9.5\%) buen ajuste, en 6 (28.6\%) aceptable, en 2 (9.5\%) malo y en ninguna muy malo (véase Tabla 7). 
Tabla 7

Correlaciones policóricas y contraste de la normalidad bivariada en mujeres

\begin{tabular}{|c|c|c|c|c|c|c|c|}
\hline \multicolumn{3}{|c|}{ Correlaciones policóricas } & \multicolumn{5}{|c|}{ Bondad de ajuste } \\
\hline Ítems & $r_{P C}[\mathrm{IC} 95 \%]$ & $E E$ & $\chi^{2}$ & $p^{*}$ & $\chi^{2} / g l$ & RMSEA & $p^{* *}$ \\
\hline RAS2 y RAS1 & $.862[.831, .893]$ & .016 & 24.099 & .063 & 1.607 & .038 & 1 \\
\hline RAS3 y RAS1 & $.816[.777, .855]$ & .020 & 11.327 & .729 & 0.755 & $<.001$ & 1 \\
\hline RAS3 y RAS2 & $.822[.785, .859]$ & .019 & 26.063 & .037 & 1.738 & .041 & 1 \\
\hline RAS4 y RAS1 & $-.633[-.702,-.564]$ & .035 & 36.674 & .001 & 2.445 & .058 & .999 \\
\hline RAS4 y RAS2 & $-.667[-.730,-.604]$ & .032 & 41.405 & $<.001$ & 2.760 & .064 & .995 \\
\hline RAS4 y RAS3 & $-.705[-.764,-.646]$ & .030 & 27.798 & .023 & 1.853 & .044 & 1 \\
\hline RAS5 y RAS1 & $.811[.772, .850]$ & .020 & 19.882 & .177 & 1.325 & .027 & 1 \\
\hline RAS5 y RAS2 & $.810[.771, .849]$ & .020 & 23.937 & .066 & 1.596 & .037 & 1 \\
\hline RAS5 y RAS3 & $.855[.824, .886]$ & .016 & 11.251 & .735 & 0.750 & $<.001$ & 1 \\
\hline RAS5 y RAS4 & $-.696[-.753,-.639]$ & .029 & 49.887 & $<.001$ & 3.326 & .073 & .972 \\
\hline RAS6 y RAS1 & $.753[.700, .806]$ & .027 & 6.258 & .975 & 0.417 & $<.001$ & 1 \\
\hline RAS6 y RAS2 & $.802[.759, .845]$ & .022 & 18.729 & .226 & 1.249 & .024 & 1 \\
\hline RAS6 y RAS3 & $.801[.758, .844]$ & .022 & 19.012 & .213 & 1.267 & .025 & 1 \\
\hline RAS6 y RAS4 & $-.706[-.767,-.645]$ & .031 & 23.154 & .081 & 1.544 & .036 & 1 \\
\hline RAS6 y RAS5 & $.805[.762, .848]$ & .022 & 12.581 & .635 & 0.839 & $<.001$ & 1 \\
\hline RAS7 y RAS1 & $-.520[-.598,-.442]$ & .040 & 16.538 & .347 & 1.103 & .015 & 1 \\
\hline RAS7 y RAS2 & $-.523[-.603,-.443]$ & .041 & 33.018 & .005 & 2.201 & .053 & 1 \\
\hline RAS7 y RAS3 & $-.518[-.598,-.438]$ & .041 & 43.987 & $<.001$ & 2.932 & .067 & .991 \\
\hline
\end{tabular}




\begin{tabular}{cccccccc}
\hline \multicolumn{3}{c}{ Correlaciones policóricas } & \multicolumn{6}{c}{ Bondad de ajuste } \\
\hline Ítems & $r_{P C}[$ IC 95\%] & $E E$ & $\chi^{2}$ & $p^{*}$ & $\chi^{2} / g l$ & $R M S E A$ & $p^{* *}$ \\
& & & & & & & \\
\hline RAS7 y RAS4 & $.579[.501, .657]$ & .040 & 58.316 & $<.001$ & 3.888 & .082 & .903 \\
RAS7 y RAS5 & $-.538[-.614,-.462]$ & .039 & 38.382 & .001 & 2.559 & .060 & .998 \\
RAS7 y RAS6 & $-.526[-.608,-.444]$ & .042 & 34.266 & .003 & 2.284 & .055 & .999 \\
\hline
\end{tabular}

*Ajuste a una distribución bivariada normal. Grados de libertad $(g l)=15$. ** RMSEA $\leq .05$.

Las soluciones de los cuatro modelos jerarquizados en las dos muestras fueron admisibles. Los pesos de medida fueron ligeramente mayores en mujeres que en hombres. Las diferencias de parámetros aún se marcaron más en la varianza del factor que fue mayor en mujeres que en hombres y en los residuos de medida que fueron menores en mujeres que en hombres. En el modelo sin restricciones y con restricciones en los pesos de medida, cinco de los seis índices de ajuste fueron buenos $\left(\chi^{2} / g l, G F I, A G F I, N F I\right.$ y $\left.R F I\right)$ y uno aceptable (SRMR). Al asumir normalidad multivariada en concordancia con las correlaciones policóricas, la bondad de ajuste se mantendría por la prueba chi-cuadrado de la razón de verosimilitud $(p>.05)$. En el modelo con restricciones en la varianza del factor, cuatro índices reflejaron buen ajuste (GFI, AGFI, NFI y RFI), uno aceptable (SRMR) y uno malo $\left(\chi^{2} / g l\right)$. En el modelo con restricciones en los residuos de medida, los índices variaron de buenos $(G F I, A G F I, N F I$ y $R F I)$ a aceptables $\left(\chi^{2} / g l\right.$ y $\left.S R M R\right)$. En estos dos últimos modelos, la bondad de ajuste se rechazaría por la prueba chi-cuadrado de la razón de verosimilitud al asumir normalidad multivariada en concordancia con las correlaciones policóricas $(p<.001)$ (véase Tabla 5). Bajo este supuesto, todos los parámetros serían 
significativos por la prueba de percentiles corregidos de sesgo en ambas muestras en los cuatro modelos anidados en restricciones.

Desde los pesos de medida del modelo sin constricciones en la muestra de mujeres (véase Figura 4), la consistencia compuesta por coeficiente rho de Joreskog fue buena ( $\rho=$ $.83)$ y el promedio de la varianza extraída fue mayor que $.5(\mathrm{AVE}=.72)$. Desde los pesos de medida del modelo sin constricciones en la muestra de hombres (véase Figura 5), la consistencia compuesta por coeficiente rho de Joreskog fue aceptable $(\rho=.78)$ y el promedio de la varianza extraída fue mayor que .5 $(\mathrm{AVE}=.62)$.

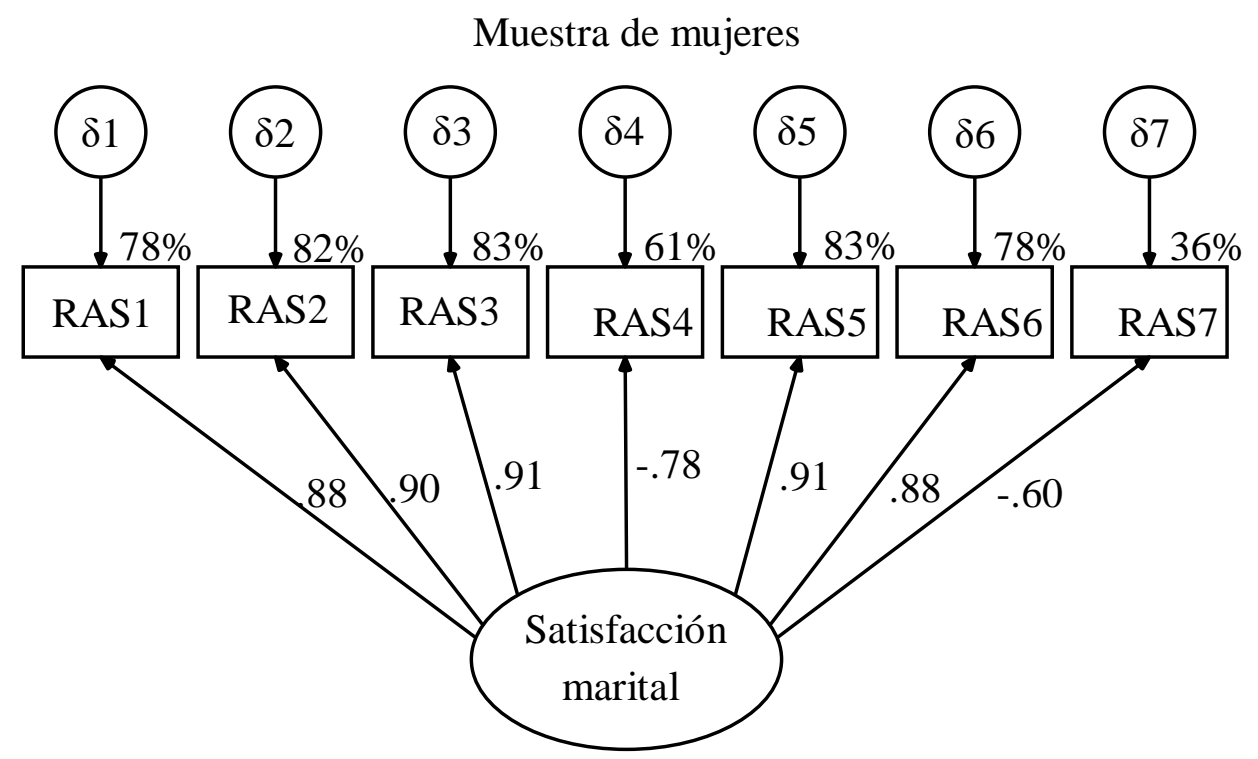

Figura 4. Modelo de un factor con siete indicadores con los coeficientes estandarizados estimados por Mínimos Cuadrados No Ponderados de escala en la muestra de mujeres. 


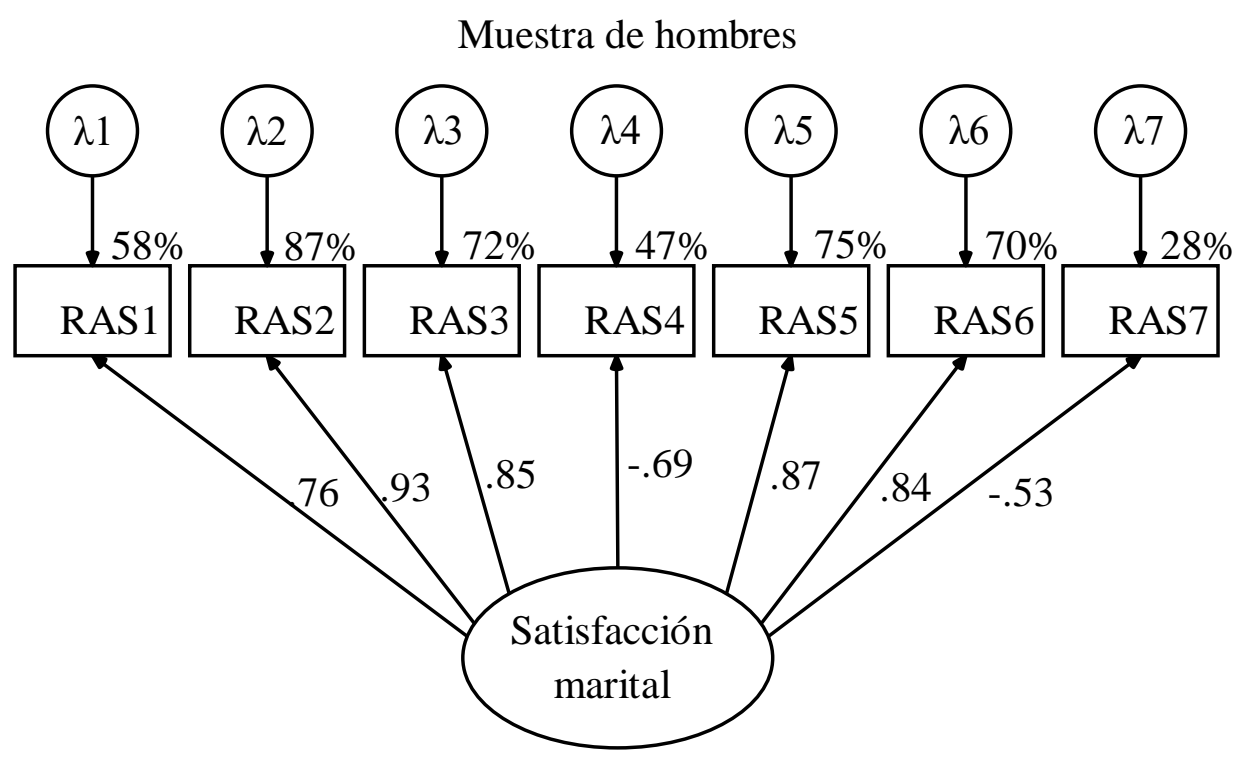

Figura 5. Modelo de un factor con siete indicadores con los coeficientes estandarizados estimados por Mínimos Cuadrados No Ponderados de escala en la muestra de hombres.

Fue mayor la bondad de ajuste del modelo modificado, esto es, con la correlación entre los residuos del ítem inverso 4 “ ¿con qué frecuencia desea no haberse implicado con su pareja?” ( 84$)$ y del ítem inverso 7 “cuántos problemas hay en su relación de pareja?” (ס6). Al ser especificado sin constricciones entre los dos sexos y con constricciones en los pesos de medida, los seis índices fueron buenos; y si se asumiera normalidad multivariada, en concordancia con las correlaciones policóricas, la bondad de ajuste se mantendría por la prueba chi-cuadrado de la razón de verosimilitud. Al ser especificado con restricciones anidadas en la varianza del factor y en los residuos de medida, los índices variaron de buenos a aceptables, pero la bondad de ajuste se rechazaría por la prueba chi-cuadrado de la razón de verosimilitud, si se asumiera normalidad multivariada. Bajo este supuesto, todos los parámetros serían significativos por la prueba de percentiles corregidos de sesgo en 
ambas muestras en los cuatro modelos anidados en restricciones y el incremento en la bondad de ajuste sería estadísticamente significativo $(p<.001)$ (véase Tabla 5).

\section{Discusión}

Este apartado se inicia con la discusión de los resultados en base a la aplicación del método elegido y se sigue con la discusión de los resultados en base a los cuatro objetivos enunciados y a las correspondientes hipótesis formuladas: estudiar la distribución, consistencia interna y discriminación de los ítems de la RAS, con la expectativa de peores propiedades en los dos ítems inversos; estimar la consistencia interna de la escala, con la expectativa de una consistencia interna buena (de .80 a .89) o incluso excelente ( $\geq .90$ ), al usarse un método más adecuado a la escala de medida ordinal de los ítems para su estimación que en estudios anteriores; comprobar si hay una convergencia de criterios empíricos para determinar el número de factores en un factor; y validar el modelo de un factor con siete indicadores y su invarianza entre ambos sexos, con la expectativas de índices de ajuste buenos o al menos adecuados.

\section{Sobre aspectos metodológicos del estudio}

En los estudios instrumentales, ya sea de desarrollo de una escala o de validación, se recomienda el empleo de muestras probabilísticas de la población a la cual se aplica el constructo medido (Montero \& León, 2002). En el presente estudio, la población fue la de personas casadas, en unión libre y con relaciones de noviazgo (Hendrick, 1988; Sabatelli, 2009). En la muestra no se incluyó personas con relaciones de noviazgo, lo que constituye una limitante del estudio. La población quedó reducida a parejas que viven juntas (casadas o en unión libre). 
Dentro del muestreo probabilístico, el muestreo estratificado y el muestreo aleatorio simple son las dos técnicas más recomendadas para garantizar la ausencia de sesgos y representatividad de la muestra recolectada (Breakwell et al., 2012). Ambas técnicas implican tener un listado de todos los individuos de la población, con la agrupación de los individuos por estratos o grupos en el primer caso, para una selección aleatoria de los mismos. En ausencia de dicho listado, pero en disposición de un mapa que ubique a la población, el muestreo de rutas aleatorias es la mejor opción (Breakwell et al., 2012). Los estudios indican que la RAS es independiente del sexo y la edad, pero sí tiene relación con otras variables sociodemográficas, como el estado civil y el número de hijos (Hendrick et al., 1998). Así se podrían haber definido estratos en relación con el estado civil (casados o en unión libre). Se optó por la aleatoriedad y el producto fue una muestra equivalente a la población en porcentajes de sexos, afiliación religiosa y estado civil, media de hijos, mediana de edad y los niveles medios de escolaridad e ingresos, con lo que se logró el objetivo de aleatoriedad (casos independientes) y representatividad (Breakwell et al., 2012).

Una cuestión importante sería la presencia de un posible sesgo de muestreo por el requisito ético del consentimiento informado. Aproximadamente ocho de cada 10 participantes dieron su consentimiento. Cabe preguntarse si el motivo de rehusar participar en la muestra pudo haberla sesgado. Sin diferencia entre ambos sexos, los tres principales motivos fueron falta de tiempo o estar muy ocupado, desinterés en participar en encuestas y desconfianza en dar datos personales, lo cual no hace sospechar de posibles sesgos, sino que, por el contrario, puede asegurar la obtención de datos de calidad, al haberse eliminado de forma natural la personas que podrían haber contestado de forma desatenta y con prisas 
el cuestionario. A su vez, los dos criterios de eliminación (presencia del cónyuge y cuestionario incompleto) garantizaron la exclusión de datos dudosos o cuestionables.

Por el objetivo de contrastar la equivalencia de la estructura factorial ente ambos sexos, inicialmente se planificó realizar un muestreo estratificado proporcional a la población en la variable de sexo, alternando la colecta de una mujer y un hombre, pero la tasa de rechazo fue diferencial. El 88\% de la mujeres consintieron y $77 \%$ de los hombres. Al lograrse una equivalencia estadística en la frecuencia de participantes de ambos sexos, se renunció al objetivo de obtener el mismo número de mujeres y de hombres en la muestra, ya que esta equivalencia estadística de frecuencia garantiza que no exista un sesgo femenino en la composición de la muestra.

El método de análisis se adecuó a la naturaleza ordinal de los datos tanto en la descripción de la distribución de los ítems y el estudio de sus propiedades de discriminación, consistencia interna y validez estructural, con el uso de la mediana, rango semiintercuartílico, coeficiente intercuartílico de asimetría, el coeficiente percentílico de curtosis, la prueba $U$ de Mann-Whitney, la correlación poliserial corregida y el coeficiente alfa ordinal (calculado sin el ítem), como en el contraste del modelo de un factor y su invarianza entre ambos sexos y el cálculo de la consistencia interna de los factores, con el uso el método de Mínimos Cuadrados No Ponderados aplicado sobre la matriz de correlaciones policóricas, el coeficiente alfa ordinal, el coeficiente $\rho$ de Joreskog y el promedio de la varianza extraída (Basto \& Pereira, 2012; Costello \& Osborne, 2005; Elosua \& Zumbo, 2008; Fornell \& Larker, 1981; Valdivieso, 2013). Además se siguió la recomendación de determinar el número de factores por la convergencia de los criterios 
más objetivos y precisos, como el análisis paralelo de Horn, la correlación parcial promedio mínima de Velicer y el análisis de coordenadas óptimas (Courtney, 2013).

Se recomienda el uso de las correlaciones policóricas con variables ordinales para evitar una subestimación de la relación lineal (Basto \& Pereira, 2012; Garrido et al., 2013; Hoffmann et al., 2013; Holgado et al., 2008; Olsson, 1979; Pearson, 1900); no obstante, existen dudas al respecto, por lo restrictivo de los supuestos (normalidad bivariada) y las dificultades para estimar las correlaciones policóricas (ajuste de un modelo de distribución continua normal a cada variable ordinal) (Yule, 1912), siendo las estimaciones inestables con tamaños de muestra pequeños $(<200)$ y claro incumplimiento de los supuestos distribucionales (Ekström, 2009; Ferrando \& Lorenzo-Seva, 2014).

Debe señalarse que las muestras usadas en el presente estudio fueron mayores de 350 participantes, con lo que se puede afirmar que las muestras no fueron pequeñas, especialmente al considerar las magnitudes altas de las cargas factoriales (Lloret et al., 2014). Se usó el método de Dos Pasos de Máxima Verosimilitud para estimar las correlaciones policóricas, que no generó ningún problema de convergencia, como es usual (Olsson, 1979). Los errores estándar de las correlaciones policóricas fueron menores o iguales que .05 , las estimaciones con un intervalo de confianza de $95 \%$ quedaron dentro del rango admisible $[-1,1]$ y las matrices de correlaciones fueron definidas positivas tanto en la muestra conjunta como en las muestras de mujeres y hombres, lo que indica que las estimaciones fueron estables. En términos generales, el ajuste del modelo de normalidad bivariada se sostuvo bien en la muestra de mujeres y de forma aceptable en la muestra de hombres y en la conjunta, considerando el residuo de aproximación cuadrático medio, el cociente entre el estadístico chi-cuadrado y sus grados de libertad, e incluso la prueba chi- 
cuadrado. Las correlaciones del ítem 4 con los ítems 5 y 7 fueron los dos casos con peor ajuste al supuesto de normalidad bivariada en las tres muestras. Al observarse una subestimación de la asociación lineal con el uso de las correlaciones de Pearson (que requieren variables numéricas con distribución normal) en comparación con las correlaciones policóricas, con una diferencia media de casi una décima, y al considerarse las propiedades de estabilidad y un aceptable cumplimiento de supuestos en las estimaciones de las correlaciones policóricas, se juzgó como mejor opción el uso de las correlaciones policóricas. Cabe señalar que existen otras opciones con supuestos menos restrictivos, como el coeficiente de correlación empírico de Ekström (2009) o la estimación bayesiana basada en el criterio del máximo a posteriori y con una distribución a priori moderadamente restrictiva (Choi et al., 2010). Esta última opción se está trabajando en la actualización del programa Factors (Ferrando \& Lorenzo-Seva, 2014), pero ninguna de las dos opciones está disponible en los programas R y LISREL, usados en este estudio.

Para determinar el número de factores se usó la matriz de correlaciones completa (modelo de análisis de componentes principales), como está recomendado (Basto \& Pereira, 2012; Courtney, 2013; Lloret et al., 2014). Si el análisis paralelo de Horn y el de coordenadas óptimas se realiza desde la matriz de correlaciones policóricas reducida (modelo factorial), se produce una sobrestimación de factores, como es usual (Basto \& Pereira, 2012). El número de factores sería dos. Extraídos por Mínimos Cuadrados No Ponderados y tras la rotación Promax, el primer factor quedaría configurado por los cinco ítems directos con una consistencia interna excelente y el segundo factor por los dos ítems inversos con la consistencia interna aceptable. La correlación entre ambos factores sería muy alta y negativa. El segundo factor tendría un número insuficiente de indicadores y un 
autovalor menor que uno. La estructura carecería de sustantividad teórica y mostraría dos factores de difícil distinción por su alta correlación. La distinción se basaría en el grado de dificultad comprensiva de los ítems, al ser los ítems inversos más difíciles de comprender, sobre todo para las personas de baja escolaridad (Lindwall et al., 2012).

Existen en México dos traducciones al español de la RAS, una realizada por Moral (2008) por el método de traducción reversa, en la cual se introdujeron modificaciones para hacerla más específica a la relación marital, y que posteriormente fue usada en el estudio de Sáenz (2014), y otra realizada por Oropeza et al. (2010). En el presente estudio se empleó la traducción original sin modificaciones hecha por Moral (2008), quien siguió las indicaciones metodológicas del Grunwald y Goldfarb (2006). Al emplearse esta versión sin las modificaciones para hacerla más específica a la relación marital, el contenido de la RAS en el presente estudio fue aún más fiel al original de Hendrick (1988) que en los estudios de Moral (2008) y Sáenz (2014).

\section{Sobre los resultados desde los objetivos enunciados e hipótesis formuladas}

En relación con el primer objetivo del estudio de las propiedades psicométricas de los siete ítems que integran la RAS, se esperaba que los siete ítems fuesen consistentes y discriminativos, siendo los dos ítems inversos los más débiles en consistencia interna y discriminación.

Conforme con la expectativa (Moral, 2008; Oropeza et al., 2010), se observó que los dos ítems inversos fueron los más débiles en las propiedades de consistencia interna y discriminación en comparación con los cinco ítems directos; no obstante, ambas propiedades fueron lo suficientemente buenas en los dos ítems inversos para ser retenidos. El ítem 4 superó el criterio de discriminación (diferencia significativa con una probabilidad 
menor que .001) y los tres criterios de consistencia interna (rPS[ítem, test-ítem] $>.5, \alpha$ ordinal [test - item] $<\alpha$ ordinal [test] y $\lambda>.5$ ); y el ítem 7 también superó todos los criterios, salvo uno de consistencia interna ( $\alpha$ ordinal [test - item] $<\alpha$ ordinal [test]). La mayor debilidad de los dos ítems inversos debe atribuirse a la dificultad comprensiva que puede generar su redacción en sentido contrario al rasgo evaluado (Lindwall et al., 2012; Sauro \& Lewis, 2011; Wu, 2008). El hecho de que la correlación policórica con peor cumplimiento del supuesto de normalidad bivariada fuese entre los dos ítems inversos en las tres muestras, probablemente se deba a los errores de respuestas entre las personas con más limitaciones en sus capacidades de comprensión verbal. Precisamente, este error de carácter metodológico podría explicar un segundo factor subyacente o una correlación entre los residuos de medida de ambos ítems (Lindwall et al., 2012; Sauro \& Lewis, 2011; Wu, 2008), el cual fue considerado para mejorar el ajuste del modelo. Aunque carece de sustantividad teórica, sí está basado en un aspecto metodológico inherente a la RAS.

En relación con el segundo objetivo de estimar la consistencia interna entre los siete ítems de la RAS, se esperaba una consistencia interna buena por la alfa de Cronbach (.80 $\leq$ $\alpha<.90$ ), pudiendo ser ésta incluso excelente al emplearse el coeficiente alfa ordinal, que es más adecuado a la escala ordinal de medida de los ítems ( $\alpha$ ordinal $\geq .90)$. La consistencia interna obtenida a través del coeficiente alfa ordinal fue excelente en la muestra conjunta, en la muestra de mujeres y en la de hombres. Conforme con la expectativa (Elosua \& Zumbo, 2008), la consistencia interna fue mayor que la estimada por el alfa de Cronbach tanto en comparación con las estimaciones dentro de la presente muestra como en comparación con el valor promedio reportado por Graham et al. (2011) en su estudio de meta-análisis ( $\alpha$ de Cronbach $=.87$, IC 95\%: .86, .88). Debe señalarse que, al ser estimada 
por el alfa de Cronbach, los valores obtenidos quedaron ligeramente por encima del intervalo medio de Graham et al. (2011), por lo que el patrón de respuesta a los ítems fue muy homogéneo dentro de cada participante en este estudio. Si se estimara por el coeficiente rho de consistencia compuesta de Jöreskog sería buena en la muestra de mujeres y en la conjunta, pero sólo aceptable en la de hombres. Estos valores serían menores que los obtenidos con el coeficiente alfa ordinal y con el coeficiente de Cronbach en la presente muestra y el promedio reportado con el alfa de Cronbach por Graham et al. (2011). Con el coeficiente alfa ordinal se logra una estimación más alta y, según el trabajo de Elosua y Zumbo (2008), ajustada a la escala ordinal de los ítems. Es importante remarcar que la consistencia interna excelente es congruente con la unidimensionalidad de la escala (Tavakol \& Dennick, 2011); asimismo, cabe destacar el hecho de que el promedio de varianza extraída sea mayor que .50 en las tres muestras, lo que proporciona validez interna al concepto de un factor (Fornell \& Larker, 1981).

En relación con el tercer objetivo de determinar el número de factores por la convergencia de criterios matemáticos empíricos, se esperaba una convergencia en un factor. Conforme a la expectativa, la convergencia de los tres criterios matemáticos empíricos más precisos (Courtney, 2013), el análisis paralelo de Horn (percentil 95), la media mínima de las correlaciones parciales al cuadrado de Velicer y el análisis de coordenadas óptimas, fue en uno. Debido a esta convergencia, debe ser fácil reproducir esta conclusión en otros estudios, incluso empleando un criterio menos preciso, como es el criterio de Kaiser. Un modelo de dos factores correlacionados, basado en el aspecto metodológico de los ítems directos e ítems inversos (Sáenz, 2014), carece de sustento teórico y los dos factores serían redundantes tener una relación muy alta entre sí. En todo 
caso se podría considerar la correlación entre los residuos de medida de los dos ítems inversos para mejorar el ajuste, como se hizo en el presente estudio.

En relación con el cuarto objetivo de validar el modelo de un factor y contrastar su invarianza entre ambos sexos, se esperaba que la estructura de un factor tuviera un ajuste a los datos entre aceptable y buena, así como unas propiedades de invarianza entre ambos sexos aceptables o buenas, aunque la correlación entre los residuos de medida de los ítems inversos 4 y 7 pudiese mejorar el ajuste.

Conforme con las expectativas, el ajuste del modelo de un factor tuvo unas propiedades buenas en términos generales y estas mejoraron al introducir una corrección en relación con la posible existencia de un segundo factor, la correlación entre los residuos de los dos ítems inversos (Sáenz, 2014). Los errores al responder de los participantes, que se confunden con la direccional de los dos ítems inversos o que adoptan una posición ambigua en su respuesta ante la duda que les generan, crean una covarianza que no posee un significado teórico, sino meramente es un efecto del diseño de la escala (Sauro \& Lewis, 2011). Por lo tanto, la modificación introducida no invalida el modelo unidimensional, sino que reconoce que la asunción de independencia entre residuos no es estricta en la RAS, al haber un efecto de error metodológico en las respuestas, atribuible a los dos ítems inversos (Iraurgi et al., 2009; Wu, 2008).

Las propiedades de invarianza del modelo de un factor entre ambos sexos son muy aceptables, especialmente el modelo corregido con la inclusión de la correlación entre los errores de medida de los dos ítems inversos. La invarianza es más estricta a nivel de pesos de medida, y menos estricta a nivel de varianza del factor y residuos de medida. En las mujeres el modelo tiene una validez interna ligeramente mayor que en los hombres. Estas 
propiedades aceptables de invarianza entre ambos sexos previamente han sido observadas en la escala de engrandecimiento marital (O'Rourke \& Cappeliez, 2001) y en la de ajuste diádico (South et al., 2009). Parece que mujeres y hombres tienen un patrón muy similar a la hora de evaluar su relación de pareja, probablemente determinado por valores y representaciones sociales que estructuran su evaluación o juicio (López \& Rodríguez, 2008). En la sociedad latina es deseable y da prestigio social estar felizmente casado y que esta relación dure toda la vida (Armenta et al., 2014).

En relación con el quinto objetivo, el modelo de dos factores de Rask et al. (2010) como posible modelo alternativo al unifactorial no fue apoyado por los criterios empíricos para determinar el número de factores. Tampoco se configuraría si se realizara un análisis exploratorio, extrayendo dos factores desde la matriz de correlaciones policóricas por el método de Mínimos Cuadrados No Ponderados y rotando la matriz factorial por el método Promax. En este caso se configuraría un primer factor con los cinco ítems directos y consistencia interna excelente ( $\alpha$ ordinal $=.95$ ) y un segundo factor con los dos ítems inversos y consistencia interna aceptable $(\alpha$ ordinal $=.73$ ), siendo la correlación entre ambas factores alta $(r=-.61)$, lo que se asemeja al resultado de Sáenz (2014). Si se especificara el modelo de dos factores de Rask et al. (2010) y la función de discrepancia fuese estimada por Mínimos Cuadrados No Ponderados, la correlación entre los dos factores sería unitaria $(r=-.91$, IC 95\%: $-.94,-.89, p=.001$ por PCS), por lo que este modelo de dos factores (amor instrumental y amor romántico) no es sustentado, al reflejar la correlación unitaria un único factor. Al estar el modelo basado en un estudio exploratorio hecho en Suecia con una muestra de tamaño menor que 200 participantes y no haberse replicado este resultado en este país u otros países, no se contrastó como posible hipótesis. 


\section{Conclusiones}

En conclusión, en la población de parejas que viven juntas (casadas o en unión libre) de la ciudad de Monterrey en México, los siete ítems de la RAS fueron consistentes y discriminativos, incluyendo los dos ítems inversos, por lo que deben ser retenidos. La consistencia interna entre los siete ítems fue excelente tanto en la muestra conjunta como en las muestras de ambos sexos al ser estimada por el coeficiente alfa ordinal, el cual logra la estimación más alta. El número de factores fue uno con base en la convergencia de tres criterios matemáticos empíricos (análisis paralelo de Horn, correlación parcial promedio mínima de Velicer y análisis de coordenadas óptimas) aplicados sobre la matriz de correlaciones policóricas completa. El modelo de un factor con la correlación entre los residuos de los dos ítems inversos tuvo un buen ajuste a los datos y unas propiedades de invarianza entre ambos sexos muy aceptables.

Se recomienda el uso de la RAS en población de parejas que viven juntas (casadas o en unión libre) por su brevedad, consistencia interna excelente y validez de constructo, como muestra el presente estudio que tiene como fortalezas el empleo de un muestreo probabilístico y unos análisis adecuados al nivel de medida de las variables. Aparte de la consistencia interna sería importante estimar la estabilidad temporal a corto plazo (2 semanas), medio plazo ( 2 meses) y largo plazo ( 2 o más años). Se hipotetiza una estabilidad temporal alta a corto y medio plazo $(r \leq .7)$. Hendrick et al. (1998) reportaron una estabilidad alta de la puntuación total de la RAS en un intervalo de seis a siete semanas entre estudiantes universitarios $(r=.85, p<.001)$; asimismo, la escala resulta ser estable en población clínica de personas que viven con una enfermedad neurológica progresiva (McCabe \& O’Connor, 2013). No obstante, la estabilidad temporal a largo plazo pudiera 
ser menor que .7, debido a tasas de deterioro en la satisfacción con la relación diferenciales entre parejas estables e inestables (Hirschberger et al., 2009). Finalmente, se sugiere la replicación del estudio en población clínica, esto es, parejas que acuden a tratamiento por desajuste, infelicidad o malos tratos, así como en parejas que no viven juntas (noviazgo).

Como limitación del presente estudio debe señalarse que su generalización sólo es válida para la población de parejas que viven juntas (casadas o en unión libre) de la ciudad de Monterrey en México. Así se previene acerca de su generalización. En otras poblaciones urbanas de parejas que viven juntas de México o de países económica y culturalmente afines, las presentes conclusiones deben manejarse como hipótesis. Una población rural o una población clínica de parejas que viven juntas (casadas o en unión libre), así como una población de novios pueden diferir más en sus características, por lo que las presentes conclusiones servirían como datos de comparación. 


\section{Referencias}

American Psychological Association (2002). Ethical principles of psychologists and code of conduct. American Psychologist, 57, 1060-1073. doi:10.1037/0003066X.57.12.1060

Anderson, J. R., Van Ryzin, M. J., \& Doherty, W. J. (2010). Developmental trajectories of marital happiness in continuously married individuals: A group-based modeling approach. Journal of Family Psychology, 24, 587-596. doi:10.1037/a0020928.

Armenta, C., Sánchez-Aragón, R., \& Díaz Loving, R. (2014). Efectos de la cultura sobre las estrategias de mantenimiento y satisfacción marital. Acta de Investigación Psicológica, 4, 1572-1584. doi:10.1016/S2007-4719(14)70394-1

Basto, M., \& Pereira, J. M. (2012). An SPSS R-Menu for ordinal factor analysis. Journal of Statistical Software, 46(4), 1-22. Recuperado el 11 de septiembre de 2015, de http://www.jstatsoft.org/v46/i04/paper

Becerra, S., Roldán, W., \& Flores, W. (2012). Factores de enriquecimiento, mantenimiento, comunicación y bienestar en la relación marital. Revista de Psicología GEPU, 3(1), 3762.

Bowen, N. K., \& Guo, S. (2011). Structural equation modeling. New York, NY: Oxford University Press.

Breakwell, G. M., Smith, J. A., \& Wright, D. B. (2012). Research methods in psychology. London, UK: Sage.

Byrne, B. M. (2010). Structural equations with AMOS: Basic concepts, applications, and programming ( $2^{\mathrm{a}}$ ed.). New York, NY: Routledge. 
Canales, C. I. (2011). Perfil de la víctima de la violencia conyugal con respecto al funcionamiento familiar, la satisfacción marital y las actitudes ante el agravio (Tesis de Maestría, Universidad de Montemorelos, México). Recuperada de http://dspace.biblioteca.um.edu.mx/jspui/bitstream/123456789/197/1/Tesis\%20Mae str\%C3\%ADa\%20\%20Carmen\%20Canales.pdf

Cañetas, E., Rivera-Aragón, S., \& Díaz-Loving, R. (2000). Desarrollo de un instrumento multidimensional de satisfacción marital. En AMEPSO (Ed.), La psicología social en México. Vol. VIII (pp. 266-274). Ciudad de México: AMEPSO.

Carlson, R. G., Barden, S. M., Daire, A. P., \& Greene, J. (2014). Influence of relationship education on relationship satisfaction for low-income couples. Journal of Counseling \& Development, 92, 418-427. doi:10.1002/j.1556-6676.2014.00168.x

Cassepp, V., \& Pasquali, L. (2011). Características psicométricas da Relationship Assessment Scale. Psico-USF, 16, 255-264. doi:10.1590/S141382712011000300002.

Chiorri, C., Day, T., \& Malmberg, L.-E. (2014). An approximate measurement invariance approach to within-couple relationship quality. Frontiers in Psychology, 5, 983. doi:10.3389/fpsyg.2014.00983

Choi, J., Peters, M., \& Mueller, R. O. (2010). Correlational analysis of ordinal data: From Pearson’s r to Bayesian polychoric correlation. Asia Pacific Education Review, 11, 459-466. doi:10.1007/s12564-010-9096-y

Costello, A. B., \& Osborne, J. (2005). Best practices in exploratory factor analysis: Four recommendations for getting the most from your analysis. Practical Assessment 
Research \& Evaluation, 10(7), 1-9. Recuperado el 24 de junio de 2015, de http://pareonline.net/getvn.asp?v=10\&n=7

Courtney, M. G. R. (2013). Determining the number of factors to retain in EFA: Using the SPSS R-Menu v2.0 to make more judicious estimations. Practical Assessment Research and Evaluation, 18(8), 24-57. Recuperado el 24 de junio de 2015, de http://pareonline.net/getvn.asp? $\mathrm{v}=18 \& \mathrm{n}=8$

Crane, D. R., Middleton, K. C., \& Bean, R. A. (2000). Establishing criterion scores for the Kansas marital satisfaction scale and the revised dyadic adjustment scale. American Journal of Family Therapy, 28, 53-60. doi:10.1080/019261800261815

Custer, L. (2009). Marital satisfaction and quality. En H. T. Reis, \& S. Sprecher (Eds.), Encyclopedia of human relationships (pp. 1030-1034). Thousand Oaks, CA: Sage. doi:10.4135/9781412958479

Dávila, J., Karney, B. R., Hall, T. W., \& Bradbury, T. N. (2003). Depressive symptoms and marital satisfaction: Within-subject associations and the moderating effects of gender and neuroticism. Journal of Family Psychology, 17, 557-570. doi:10.1037/0893-3200.17.4.557

Díaz-Loving, R., \& Rivera-Aragón, S. (2002). La psicología del amor: Una visión integral de la relación de pareja. Ciudad de México: Miguel Ángel Porrúa.

Dinkel, A., \& Balck, F. (2005). An evaluation of the German relationship assessment scale. Swiss Journal of Psychology, 64, 259-263. doi:10.1024/1421-0185.64.4.259

Ekström, J. (2009). Contributions to the theory of measures of association for ordinal variables. Acta Universitatis Upsaliensis, 50, 1-32. Recuperado el 15 de septiembre de 2015, de https://uu.diva-portal.org/smash/get/diva2:210896/FULLTEXT01.pdf 
Elosua, P., \& Zumbo, B. (2008). Coeficientes de fiabilidad para escalas de respuesta categórica ordenada. Psicothema, 20, 896-901.

Ferrando, P. J., \& Lorenzo-Seva, U. (2014). El análisis factorial exploratorio de los ítems: algunas consideraciones adicionales. Anales de Psicología, 30, 1170-1175. doi:10.6018/analesps.30.3.199991.

Flores, M. M. (2011). Comunicación y conflicto: ¿qué tanto impactan en la satisfacción marital? Acta de Investigación Psicológica, 1(2), 216-232.

Fornell, C., \& Larker, D. F. (1981). Evaluating structural equation models with unobservable variables and measurement error. Journal of Marketing Research, 18, 39-50. doi: $10.2307 / 3151312$

Garrido, L. E., Abad, F. J., \& Ponsoda, V. (2013). A new look at Horn's parallel analysis with ordinal variables. Psychological Methods, 18, 454-474. doi:10.1037/a0030005

Gottman, J. M., \& Notarius, C. I. (2002). Marital research in the 20th century and a research agenda for the 21st century. Family Process, 41, 159-197. doi:10.1111/j.1545-5300.2002.41203.x

Graham, J. M., Diebels, K. J., \& Barnow, Z. B. (2011). The reliability of relationship satisfaction: A reliability generalization meta-analysis. Journal of Family Psychology, 25, 39-48. doi:10.1037/a0022441.

Grunwald, D., \& Goldfarb, N. M (2006). Back translation for quality control of informed consent forms. Journal of Clinical Research Best Practices, 2(2), 1-6. Recuperado el 12 de julio de 2015, de www.translationdirectory.com/article1043.htm

Hendrick, S. S. (1988). A generic measure of relationship satisfaction. Journal of Marriage and the Family, 50, 93-98. doi:10.2307/352430 
Hendrick, S. S., Dicke, A., \& Hendrick, C. (1998). The relationship assessment scale. Journal of Social and Personal Relationships, 15, 137-142. doi: $10.1177 / 0265407598151009$.

Hirschberger, G., Srivastava, S., Marsh, P., Cowan, C. P., \& Cowan, P. A. (2009). Attachment, marital satisfaction, and divorce during the first fifteen years of parenthood. Personality Relationship, 16, 401-420. doi:10.1111/j.14756811.2009.01230.x

Hoffmann, A. F., Stover, J. B., De la Iglesia, G., \& Fernández, M. (2013). Correlaciones policóricas y tetracóricas en estudios factoriales exploratorios y confirmatorios. Ciencias Psicológicas, 7(1), 151-164.

Holgado, F. P., Chacón, S., Barbero, I., \& Vila, E. (2008). Polychoric versus Pearson correlations in exploratory and confirmatory factor analysis of ordinal variables. Quality \& Quantity, 44, 153-166. doi:10.1007/s11135-008-9190-y

Hudson, W. W. (1992). The WALMYR assessment scales scoring manual. Tallahassee, FL: WALMYR Publishing.

Instituto Nacional de Estadística, Geografía e Informática (2010). Principales resultados del censo de población y vivienda 2010: Nuevo León. Ciudad de México: INEGI. Recuperado el 22 de junio de 2015, de http://www.inegi.org.mx/prod_serv/contenidos/espanol/bvinegi/productos/censos/po blacion/2010/princi_result/nl/19_principales_resultados_cpv2010.pdf

Instituto Nacional de Estadística, Geografía e Informática (2011). Panorama sociodemográfico de Nuevo León. Ciudad de México: INEGI. Recuperado el 22 de junio de 2015, de 


\section{http://www.inegi.org.mx/prod serv/contenidos/espanol/bvinegi/productos/censos/po} blacion/2010/panora_socio/nl/Panorama_NL.pdf

Iraurgi, I., Sanz, M., \& Martínez, A. (2009). Adaptación y estudio psicométrico de dos instrumentos de pareja: índice de satisfacción matrimonial y escala de inestabilidad matrimonial. Revista de Investigación en Psicología, 12, 177-192.

Jackson, J. B., Miller, R. B., Oka, M., \& Henry, R. G. (2014). Gender differences in marital satisfaction: A meta-analysis. Journal of Marriage and Family, 76, 105-129. doi:10.1111/jomf.12077

James, L. R., Mulaik, S. A., \& Brett, J. M. (1982). Causal analysis: Assumptions, models and data. Beverly Hills, CA: Sage.

Joreskog, K. G., \& Sörbom, D. (2000). LISREL 8.5. User's reference guide. Chicago, IL: Scientific Software International.

Kamp, C. M., Taylor, M. G., \& Kroeger, R. A. (2008). Marital happiness and psychological well-being across the life course. Family Relations, 57(2), 1-23. doi:10.1111/j.17413729.2008.00495.x

Lavner, J. A., \& Bradbury, T. N. (2010). Patterns of change in marital satisfaction over the newlywed years. Journal of Marriage and the Family, 72, 1171-1187. doi:10.1111/j.1741-3737.2010.00757.x

Lawrence, E., Barry, R. A., Langer, A., \& Brock, R. L. (2009). Assessment of marital satisfaction. En H. T. Reis, \& S. Sprecher (Eds.), Encyclopedia of human relationships (pp. 1028-1030). Thousand Oaks, CA: SAGE Publications. doi:10.4135/9781412958479 
Li, T., \& Fung, H. H. (2011). The dynamic goal theory of marital satisfaction. Review of General Psychology, 15, 246-254. doi:10.1037/a0024694

Lindwall, M., Barkoukis, V., Grano, C., Lucidi, F., Raudsepp, L., Liukkonen, J., \& Thøgersen-Ntoumani. C. (2012). Method effects: The problem with negatively versus positively keyed items. Journal of Personality Assessment, 94, 196-204. doi:10.1080/00223891.2011.645936

Lloret, S., Ferreres, A., Hernández, A., \& Tomás, I. (2014). El análisis factorial exploratorio de los ítems: una guía práctica, revisada y actualizada. Anales de Psicología, 30, 1151-1169. doi.org/10.6018/analesps.30.3.199361

López, E., \& Rodríguez, N. (2008). Relación entre cultura del honor, celos y satisfacción en la pareja. Boletín de Psicología, 94, 7-22.

Martínez, V., Peiró, J. M., \& Ramos, J. (2001). Calidad de servicio y satisfacción del cliente: una perspectiva psicosocial. Madrid: Síntesis.

McCabe, M., \& O’Connor, E. (2013). A longitudinal study of quality of life among people living with a progressive neurological illness. Health, 5, 17-23. doi:10.4236/health.2013.56A2004.

Mendoza, L. A., Soler, E., Sainz, L., Gil, I., Mendoza, H. F., \& Pérez, C. (2006). Análisis de la dinámica y funcionalidad familiar en atención primaria. Archivos en Medicina Familiar, 8(1), 27-32.

Montero, I., \& León, O. G. (2002). Clasificación y descripción de las metodologías de investigación psicología. International Journal of Clinical and Health Psychology, 2, 503-508. 
Moral, J. (2008). Validación de la escala de valoración de la relación en población mexicana. Revista Electrónica de Metodología Aplicada, 13(1), 1-12. Recuperado el 24 de junio de 2015, de http://www.psico.uniovi.es/REMA/v13n1/vol13n1a1.pdf.

Norton, R. (1983). Measuring marital quality: A critical look at the dependent variable. Journal of Marriage and Family, 45, 141-151. doi:10.2307/351302

O'Rourke, N., \& Cappeliez, P. (2001). Marital satisfaction and marital aggrandizement among older adults: Analysis of gender invariance. Measurement and Evaluation in Counseling and Development, 34, 66-79.

Olson, D. H., Fournier, D. G., \& Druckman, J. M. (1985). ENRICH: Enriching and nurturing relationship issues, communication and happiness. En D. H. Olson, H. I. McCubbin, H. Barnes, A. Larsen, M. Muxen, \& M. Wilson (Eds.), Family inventories. Family social sciences (pp. 43-50). St. Paul, MN: University of Minnesota.

Olsson, U. (1979). Maximum likelihood estimation of the polychoric correlation coefficient. Psychometrika, 44, 443-460. doi:10.1007/BF02296207

Oropeza, R., Armenta, C., García, A., Padilla, N., \& Díaz-Loving, R. (2010). Validación de la escala de evaluación de relaciones en población Mexicana. Revista Psicología Iberoamericana, 18(2), 56-65.

Pearson, K. (1900). Mathematical contributions to the theory of evolution. VII. On the correlation of characters not quantitatively measurable. Philosophical Transitions of the Royal Society, Series A, 195, 1-47. Recuperado el 15 de septiembre de 2015, de http://www.jstor.org/stable/90764 
Pick, S., \& Andrade, P. (1988). Desarrollo y validación de la escala de satisfacción marital (ESM). Psiquiatría, 4(1), 9-20.

Rask, M., Malm, D., Kristofferzon, M. L., Roxberg, A., Svedberg, P., Arenhall, E., ... SAMMI-Study Group (2010). Validity and reliability of a Swedish version of the Relationship Assessment Scale (RAS): A pilot study. Canadian Journal of Cardiovascular Nursing, 20(1), 16-21.

Sabatelli, R. M. (2009). Satisfaction in relationships. En H. T. Reis, \& S. Sprecher (Eds.), Encyclopedia of human relationships (pp. 1398-1402). Thousand Oaks, CA: Sage. doi: $10.4135 / 9781412958479$

Sáenz, N. E. (2014). Modelo para la promoción del sexo seguro en parejas estables (Tesis de doctorado, Universidad Autónoma de Nuevo León, México). Recuperada de http://eprints.uanl.mx/3983/1/1080253625.pdf

Sauro, J., \& Lewis, J. R. (2011). When designing usability questionnaires, does it hurt to be positive? Proceedings of the SIGCHI Conference on Human Factors in Computing System, 11, 2215-2224. doi:10.1145/1978942.1979266

Schumm, W. R., Nichols, C. W., Schectman, K. L., \& Grinsby, C. C. (1983). Characteristics of responses to the Kansas marital satisfaction scale by a sample of 84 married mothers. Psychological Reports, 53, 567-572.

Schumm, W. R., Paff-Bergen, L. A., Hatch, R. C., Obiorah, F. C., Copeland, J. M., Meens, L. D., \& Bugaihis, M. A. (1986). Concurrent and discriminant validity of the Kansas marital satisfaction scale. Journal of Marriage and the Family, 48, 381-387. doi: $10.2307 / 352405$ 
Secretaría de Salud de Nuevo León (2013). Resultados por región de la primera encuesta estatal de salud y nutrición-Nuevo León 2011/2012. Monterrey: SS-NL. Recuperado el 24 de junio de $2015, \quad$ de http://www.saludnl.gob.mx/drupal/sites/default/files/encuesta_salud_y_nutricion.pd $\underline{\mathrm{f}}$

Shackelford, T. K., Besser, A., \& Goetz, A. T. (2008). Personality, martial satisfaction, and probability of marital infidelity. Individual Differences Research, 6, 13-25.

Snyder, D., Heyman, R., \& Haynes, S. N. (2009). Strategies in the assessment of couples. En J. Butcher (Ed.). Oxford handbook of personality assessment (pp. 457-484). Oxford, UK: Oxford University Press.

South, S. C., Krueger, R. F., \& Iacono, W. G. (2009). Factorial invariance of the dyadic adjustment scale across gender. Psychological Assessment, 21, 622-628. doi: $10.1037 / \mathrm{a} 0017572$

Stone, E., \& Shackelford, T. (2007). Marital satisfaction. En R. Baumeister, \& K. Vohs (Eds.), Encyclopedia of social psychology (pp. 541-545). Thousand Oaks, CA: SAGE Publications, Inc. doi:10.4135/9781412956253.n323

Tavakol, M., \& Dennick, R. (2011). Making sense of Cronbach's alpha. International Journal of Medical Education, 2, 53-55. doi:10.5116/ijme.4dfb.8dfd

Valdivieso, C. E. (2013). Efecto de los métodos de estimación en las modelaciones de estructuras de covarianzas sobre un modelo estructural de evaluación del servicio de clases. Comunicaciones en Estadística, 6(1), 21-44.

Ward, P. J., Lundberg, N. R., Zabriskie, R. B., \& Berrett, K. (2009). Measuring marital satisfaction: A comparison of the revised dyadic adjustment scale and the 
satisfaction with married life scale. Marriage and Family Review, 45, 412-429. doi:10.1080/01494920902828219

Wu, C. H. (2008). An examination of the wording effect in the Rosenberg self-esteem scale among culturally Chinese people. Journal of Social Psychology, 148, 535-551. doi:10.3200/SOCP.148.5.535-552.

Yang, Y., \& Green, S. B. (2010). A note on structural equation modeling estimates of reliability. Structural Equation Modeling: A Multidisciplinary Journal, 17, 66-81. doi:10.1080/10705510903438963

Yule, G. U. (1912). On the methods of measuring the association between two attributes. Journal of the Royal Statistical Society, 75, 579-652. doi: 10.2307/2340126 


\section{(ㄷ) (1)}

Este texto está protegido por una licencia Creative Commons $\underline{4.0}$.

Usted es libre para Compartir — copiar y redistribuir el material en cualquier medio o formato-y Adaptar el documento - remezclar, transformar y crear a partir del material - para cualquier propósito, incluso comercialmente, siempre que cumpla la condición de:

Atribución: Usted debe reconocer el crédito de una obra de manera adecuada, proporcionar un enlace a la licencia, e indicar si se han realizado cambios. Puede hacerlo en cualquier forma razonable, pero no de forma tal que sugiera que tiene el apoyo del licenciante o lo recibe por el uso que hace. 